$\mathrm{MFN}=008666$

$01 \mathrm{SID} / \mathrm{SCD}$

027316

03 INPE-7316-PRE/3211

04 CEA

$05 \mathrm{~S}$

06 as

10 Tsurutani, Bruce T.

10 Gonzalez-Alarcon, Walter Demetrio

10 Gonzalez-Alarcon, Alicia Luisa Clua

10 Tang, Frances

10 Arballo, John K.

10 okada, Masaki

12 Interplanetary origin of geomagnetic activity in the declining phase of the solar cycle

$14 \quad 21717-21733$

30 Journal of geophysical Research

31100

32 A11

$40 \mathrm{En}$

41 En

58 DGE

$61<\mathrm{PI}>$

64 Nov, <1995>

68 PRE

76 GEOFISICA ESPACIAL

83 Interplanetary magnetic field (IMF) and plasma data are compared with ground-based geomagnetic Dst and $\mathrm{AE}$ indices to determine the causes of magnetic storms, substorms, and quiet during the descending phase of the solar cycle. In this paper we focus primarily on 1974 when the $\mathrm{AE}$ index is anomalously high $(\mathrm{AE}=283 \mathrm{nT}$ ). This year is characterized by the presence of two long-lasting corotating streams associated with coronal holes. The corotating streams interact with the upstream low-velocity $(300-350 \mathrm{~km} \mathrm{~s}-1)$, high-density heliospheric current sheet (HCS) plasma sheet, which leads to field compression and $\sim 15$ to $25-n \mathrm{~T}$ hourly average values. Although the $\mathrm{Bz}$ component in this corotating interaction region (CIR) is often < $10 \mathrm{nT}$, typically the field directionality is highly variable, and large southward components have durations less than 3 hours. Thus the corotating strean/HCS plasma sheet interaction region can cause recurring moderate $(-100 \mathrm{nT}: 5$ Dst... $-50 \mathrm{nT}$ ) to weak (50 nT...Dst:5 $-25 \mathrm{nT}$ ) storms, and sometimes no significant ring current activity at all (Dst > -25 nT). Storms of major (Dst... - $100 \mathrm{nT}$ ) intensities were not associated with CIRs. Solar wind energy is transferred to the magnetosphere via magnetic reconnection during the weak and moderate storms. Because the $B$, component in the interaction region is typically highly fluctuating, the corresponding storm main phase profile is highly irregular. Reverse shocks are sometimes present at the sunward edge of the CIR. Because these events cause sharp decreases in field magnitude, they can be responsible for storm 
recovery phase onsets. The initial phases of these corotating stream-related storms are caused by the increased ram pressure associated with the HCS plasma sheet and the further density enhancement from the stream-stream compression. Although the solar wind speed is generally low in this region of space, the densities can be well over an order of magnitude higher than the average value, leading to significant positive Dst values. Since there are typically no forward shocks at 1 AU associated with the stream-stream interactions, the initial phases have gradual onsets. The most dramatic geomagnetic response to the corotating streams are chains of consecutive substorms caused by the southward components of large-amplitude Alfven waves within the body of the corotating strearns. This auroral activity has been previously named high-intensity long-duration continuous AE activity (HILDCAAs). The substorm activity is generally most intense near the peak speed of the stream where the Alfven wave amplitudes are greatest, and it decreases with decreasing wave amplitudes and stream speed. Each of the 27-day recurring HILDCAA events can last 10 days or more, and the presence of two events per solar rotation is the cause of the exceptionally high AE average for 1974 (higher than 1979). HILDCAAs often occur during the recovery phase of magnetic storms, and the fresh (and sporadic) injection of substorin energy leads to unusually long storm recovery phases as noted in Dst In the far trailing edge of the corotating stream, the IMF amplitudes become low, <3 nT, and there is an absence of large-amplitude fluctuations (Alfven waves). This is related to and causes geomagnetic quiet. There were three major (Dst... -100 nT) storms that occurred in 1974. Each was caused by a nonrecurring moderate speed stream led by a fast forward shock. The mechanisms for generating the intense interplanetary $B$, which were responsible for the subsequent intense magnetic storms was shock compression of preexisting southwardly directed $\mathrm{Bz}$ (Bs) for the two largest events and a magnetic cloud for the third (weakest) event. Each of the three streams occurred near a HCS crossing with no obvious solar optical or $X$ ray signatures. It is speculated that these events may be associated with flux openings associated with coronal hole expansions. In conclusion, we present a model of geomagnetic activity during the descending phase of the solar cycle. It incorporates storm initial phases, main phases, HILDCAAs, and geomagnetic quiet. It uses some of the recent ulysses results. We feel that this model is sufficiently developed that it may be used for predictions, and we encourage testing during the current Dhase of the solar cycle.

87 MAGNETOSFERA

87 TEMPESTADES MAGNETICAS

87 CICLO SOLAR

$90 \mathrm{~b}$

91 FDB-20000106

92 FDB-MLR 
responsible for storm recovery phase onsets. The initial phases of these corotating stream-related storms are caused by the increased ram pressure associated with the HCS plasma sheet and the further density enhancement from the stream-stream compression. Although the solar wind speed is generally low in this region of space, the densities can be well over an order of magnitude higher than the average value, leading to significant positive Dst values. Since there are typically no forward shocks at 1 AU associated with the stream-stream interactions, the initial phases have gradual onsets. The most dramatic geomagnetic response to the corotating streams are chains of consecutive substorms caused by the southward components of large-amplitude Alfven waves within the body of the corotating streams. This auroral activity has been previously named high-intensity long-duration continuous AE activity (HILDCAAs). The substorm activity is generally most intense near the peak speed of the stream where the decreases with decreasing wave amplitudes and stream speed. Each of the 27-day recurring HILDCAA events can last 10 days or more, and the presence of two events per solar rotation is the cause of the exceptionally high $\mathrm{AE}$ average for 1974 (higher than 1979). HILDCAAs often occur during the recovery phase of magnetic storms, and the fresh (and sporadic) injection of substorin energy leads to unusually long storm recovery phases as noted in Din the far trailing edge of the corotating stream, the IMF amplitudes become low, $<3 \mathrm{nT}$, and there is an absence of large-amplitude fluctuations (Alfven waves). This is related to and causes geomagnetic quiet. There were three major (Dst $-100 \mathrm{nT}$ ) storms that occurred in 1974 . Each was caused by a nonrecurring moderate speed stream led by a fast forward shock. The mechanisms for generating the intense interplanetary $B$, which were responsible for the subsequent intense magnetic storms was shock compression of preexisting southwardly directed $\mathrm{B}_{\mathcal{G}}\left(\mathrm{B}_{\mathcal{E}}\right)$ for the two largest events and a magnetic cloud for the third (weakest) event. Each of the three streams occurred near a HCS crossing with no obvious solar optical or $x$ ray signatures. It is speculated that these events may be associated with flux openings associated with coronal hole expansions. In conclusion, we present a model of geomagnetic activity during the descending phase of the solar cycle. It incorporates storm initial phases, main phases, HILDCAAs, and geomagnetic quiet. It uses some of the recent Ulysses results. We feel that this model is sufficiently developed that it may be used for predictions, and we encourage testing during the current Dhase of the solar cycle.

87 MAGNETOSFERA

87 TEMPESTADES MAGNETICAS

87 CICLO SOLAR

$90 \mathrm{~b}$

91 FDB-20000106

92 FDB-MLR 


\title{
Interplanetary origin of geomagnetic activity in the declining phase of the solar cycle
}

\author{
Bnce T. Tsurutani,' Walter D. Gonzalez, ${ }^{2}$ Alicia L. C. Gonzalez, ${ }^{2}$ Frances Tang, ${ }^{3}$ \\ John K. Arballo,' and Masaki Okada ${ }^{4}$
}

\begin{abstract}
Interplanetary magnetic field (MMF) and plasma data are compared with ground-based geomagnetic $D s t$ and $A E$ indices to determine the causes of magnetic storms, substorms, and quiet during the descending phase of the solar cycle. In this paper we focus primarily on 1974 when the $A E$ index is anomalously high $(\overrightarrow{A E}=283 \mathrm{nT})$. This year is characterized by the presence of two long-lasting corotating streams associated with coronal holes. The corotating streams interact with the upstream low-velocity (300-350 $\mathrm{km} \mathrm{s}^{-1}$ ), high-density heliospheric current sheet (HCS) plasma sheet, which leads to field compression and $\sim 15-$ to $25-n T$ hourly average values. Although the $B_{z}$ component in this corotating interaction region (CIR) is often $<-10 \mathrm{nT}$, typically the field directionality is highly variable, and large southward components have durations less than 3 hours.
\end{abstract} Thus the corotating stream/HCS plasma sheet interaction region can cause recurring moderate $(-100 \mathrm{nT} \leq D s t \leq-50 \mathrm{nT})$ to weak $(-50 \mathrm{nT} \leq D s t \leq-25 \mathrm{nT})$ storms, and sometimes no significant ring current activity at all (Dst $>-25 \mathrm{nT}$ ). Storms of major (Dst $\leq-100 \mathrm{nT}$ ) intensities were not associated with CIRs. Solar wind energy is transferred to the magnetosphere via magnetic reconnection during the weak and moderate storms. Because the $B_{z}$ component in the interaction region is typically highly fluctuating, the corresponding storm main phase profile is highly iregular. Reverse shocks are sometimes present at the sunward edge of the CIR. Because these events cause sharp decreases in field magnitude, they can be responsible for storm recovery phase onsets. The initial phases of these corotating stream-related storms are caused by the increased ram pressure associated with the HCS plasma sheet and the further density enhancement from the stream-stream compression. Although the solar wind speed is generally low in this region of space, the densities can be well over an order of magnitude higher than the average value, leading to significant positive Dst values. Since there are typically no forward shocks at $1 \mathrm{AU}$ associated with the stream-stream interactions, the initial phases have gradual onsets. The most dramatic geomagnetic response to the corotating streams are chains of consecutive substorms caused by the southward components of large-amplitude Alfvén waves within the body of the corotating streams. This auroral activity has been previously named high-intensity long-duration continuous $A E$ activity (HILDCAAs). The substorm activity is generally most intense near the peak speed of the stream where the Alfven wave amplitudes are greatest, and it decreases with decreasing wave amplitudes and stream speed. Each of the 27-day recurring HILDCAA events can last 10 days or more, and the presence of two events per solar rotation is the cause of the exceptionally high $A E$ average for 1974 (higher than 1979). HILDCAAs often occur during the recovery phase of magnetic storms, and the fresh (and sporadic) injection of substorm energy leads to unusually long storm recovery phases as noted in Dst. In the far trailing edge of the corotating stream, the IMF amplitudes become low, $<3 \mathrm{nT}$, and there is an absence of large-amplitude fluctuations (Alfven waves). This is related to and causes geomagnetic quiet. There were three major (Dst $\leq-100 \mathrm{nT}$ ) storms that occurred in 1974. Each was caused by a nonrecurring moderate speed stream led by a fast forward shock. The mechanisms for generating the intense interplanetary $B_{s}$ which were responsible for the subsequent intense magnetic storms was shock compression of preexisting southwardly directed $B_{z}\left(B_{s}\right)$ for the two largest events and a magnetic cloud for the third (weakest) event. Each of the three streams occurred near a HCS crossing with no obvious solar optical or $\mathrm{X}$ ray signatures. It is speculated that these events may be associated with flux openings associated with coronal hole expansions. In conclusion, we present a model of geomagnetic activity during the descending phase of the solar cycle. It incorporates storm initial phases, main phases, HILDCAAs, and geomagnetic quiet. It uses some of the recent Ulysses results. We feel that this model is sufficiently developed that it may be used for predictions, and we encourage testing during the current phase of the solar cycle.

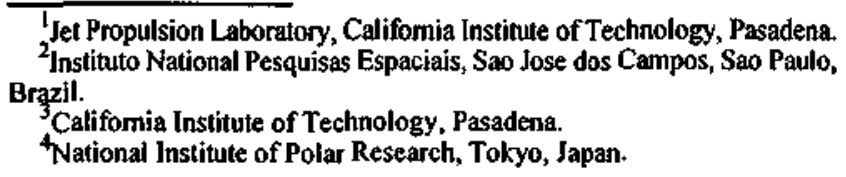




\section{Introduction}

Recently, considerable attention has focused on the interplanetary and solar causes of geomagnetic storms (here we use the Gonzalez et al. [1994] definition of a magnetic storm as an event with substantially negative $D s t$ ) that have occurted at and near solar maximum [Gonzalez and Tsurutani, 1987; Burlaga et al., 1987; Tsurutani et al., 1988, 1992; Tang et al., 1989; Gonzalez et al., 1989, 1992, 1994; Gosling et al., 1990, 1991; Hoeksema and Zhao, 1992; Crooker et al., 1992a; Farrugia et al., 1993a, b; Tsurutani and Gonzalez, 1995a]. Almost all of the large storm events were associated with fast ejecta that have impinged upon the Earth's magnetosphere. The mechanisms causing the geomagnetic activity have been shown to be compressed southward directed magnetic fields $\left(B_{s}\right)$ behind interplanetary shocks [Tsurutani et al., 1988] or southward fields within the magnetic cloud [Burlaga, et al, 1981; Klein and Burlaga, 1982] portion of the driver gases (see Gonzalez et al. [1994] for a review). Magnetic reconnection [Dungey, 196/] between the interplanetary and magnetopause fields is the energy transfer mechanism creating the storms.

During the descending phase of the solar cycie, high-velocity solar ejecta occur less frequently, and corotating high-speed streams occur more often [Feynman and $G u, 1986]$ ]. The corotating streams have been associated with polar coronal holes [Krieger et al., 1973, 1974; Timothy et al., 1975] that have extended to latitudes close to the ecliptic plane [Burlaga et al., 1978]. Because the holes are (relatively) fixed at the Sun, the high-speed streams "corotate" with the Sun's $\sim 27$ day rotation rate [Timothy et al., 1975].

It has been known for a long time [Bartels, 1938; Chapman and Bartels, 1940; Dessler and Fejer, 1963] that moderate level geomagnetic activity can occur with a $\sim 27$ day period. Clearly, it is the corotating streams [Burlaga and Lepping, 1977; Sheeley et al., 1977; Burlaga et al., 1978], the preceding heliospheric current sheet (HCS) crossings [Smith et al., 1978], or the streamstream interaction regions [Belcher and Davis, 1971; Pizzo, 1985] that are responsible for such geomagnetic activity. It is the purpose of this paper to determine what solar wind plasma and magnetic field features are responsible for geomagnetic storms, substorms, and geomagnetic quiet during the descending phase of the solar cycle when the corotating streams dominate in occurrence rate over fast ejecta. There are several reasons to expect that the cause of geomagnetic activity will be considerably different than during solar maximum. The corotating streams are not expected to have embedded magnetic clouds with intense southward fields. Also, because the plasma is continuously emitted from the coronal holes, fast forward (and reverse) shocks should only form at the corotating interaction region (CIR) boundaries [Smith and Wolfe, 1976]; for example, see Figure 13 of Belcher and Davis [1971]. Pioneer 10 and 11 observations have shown that fast forward shocks associated with corotating streams typically do not form until $\sim 1.5 \mathrm{AU}$ from the Sun, and reverse shocks not until $-2.5 \mathrm{AU}$ [Smith and Wolfe, 1976]. The absence of a forward shock at the antisolar edge of a fast stream and a -27-day recurrence of the stream has been used to tdentify such corotating structures.

This paper will attempt to build on previous solar wind works which have dealt with the plasma density enhancements neat heliosphere current sheets [Belcher and Davis, 1971; Burlaga et al, 1978; Borrini et al., 1981], high-speed corotating streams adjacent to the HCS plasma sheet [Sheeley et al., 1976, 1977; Gosling et al., 1976] (these structures have been called HCS plasma sheets by Winterhalter et al. [1994]), magnetic sector

\section{SOLAR WIND - MAGNETIC STORM CORRELATIONS}

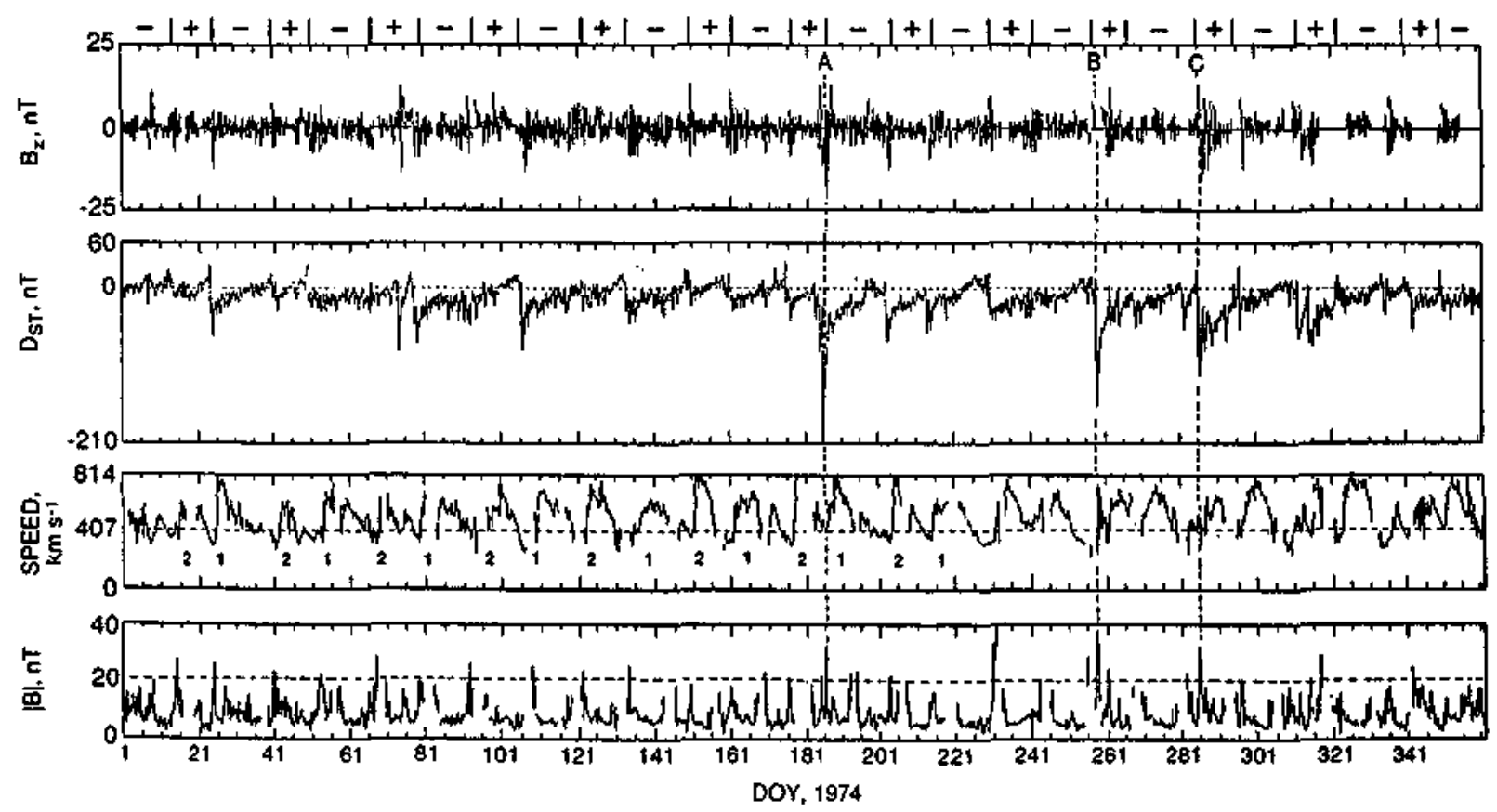

Figure 1. The IMF $B_{z}, D s t, V_{s w}$, and IMF $|B|$ for 1974 . Two sequences of corotating streams are indicated in the solar wind speed panel. These streams are responsible for moderáte to weak magnetic storms. The three largest intensity magnetic storms are called out as the A, B, and C events. These storms are caused by fast ejecta from the Sun and are not related to corotating streams. The sectors are denoted at the top of the figure. 
structures or heliospheric current sheets [Ness and Wilcox, 1964; Wilcox and Ness 1965; Smith et al., 1978], high-speed corotating streams adjacent to the HCS plasma sheet [Sheeley et al., 1976, 1977; Gosling et al, 1976], and stream-stream magnetic field compressive effects [Burlaga et al., 1977, 1978]. The purpose of this paper will be to put the above structures into context and to specifically address the nature of the interplanetary magnetic field (IMF) $B_{z}$ component, which will be shown to be the primary factor in causing geomagnetic activity/lack of activity during the descending phase of the solar cycle.

\section{Data Selection and Method of Analyses}

We have selected three years during the descending phase of solar cycle 20 for this study (1973-1975). This particular interval was chosen because of the presence of two long-lasting coronal holes that provided corotating high-speed streams in the interplanetary medium [Hansen et al., 1976; Burlaga et al., 1978; Tsurutani et al., 1982; Lindblad and Lundstedt, 1983; Lindblad, 1990]. Although all three years have been analyzed, for purposes of illustration we will focus primarily on the data from 1974. This year contains the cleanest, most unambiguous examples of recurring streams and is representative of the three years of study.

In order to perform this study, both low temporal resolution ( -1 hour) and high-resolution (seconds to minutes) magnetic field and plasma data are used. The former will be used to illustrate the large-scale timing features of the high-speed streams and HCS plasma sheet events, and the latter to study the detailed causes of the southward $B_{z}\left(B_{s}\right)$ events. We have used the OMNI l-hour averages of the interplanetary parameters for the former and have also obtained high-resolution IMP 7 and 8 plasma and magnetic field data from the National Space Science Data Center, Goddard Space Flight Center, for the latter. Because the IMP spacecraft are Earth-orbiting, there are interplanetary data gaps when the satellites enter the magnetosphere/magnetotail. These gaps unfortunately cannot be avoided. For the high-resolution data, the gaps are filled with straight lines. Dst (1 hour) and $A E(2.5 \mathrm{~min})$ indices are used to indicate geomagnetic storm activity and substorm activity, respectively. The $2.5-\mathrm{min} A E$ indices were averaged to 1 hour where appropriate. Dst has been shown to be linearly related to the total ring current particle energy by Sckopke [1966], and we use this index as a proxy for the latter. Three-hour $a p$ indices are shown as well. These indices were obtained from the World Data Center A (WDC-A), Boulder, Colorado.

We use the WDC-A uncorrected Dst indices throughout the paper. Intervals such as the initial phases of storms can be better studied without introducing pressure corrections; thus our usage of raw values. It should be noted, however, that for some main phase intervals, the uncorrected values can depart from the corrected values. Thus some storm intensities might be greater than those shown in this paper.

\section{Results}

High-Speed Corotating Streams and Associated Magnetic Field Structures

Figures 1 and 2 jllustrate hourly averages of the magnetic field and plasma data and geomagnetic indices for the year 1974. These figures are shown to give an overview of the geomagnetic activity for the entire year (high-resolution plots are available upon request from the authors). In Figure 1, from top to bottom, are the interplanetary magnetic field $B_{z}$ component in GSM coordinates, Dst, the solar wind speed, and the magnetic field magnitude. In the GSM coordinate system, $\hat{x}$ is toward the Sun, $\hat{y}$ is defined by $\hat{n} x \hat{x} /|\hat{n} x \hat{x}|$, and $\hat{z}$ forms a right-hand system. Here $\hat{n}$ is the south magnetic pole direction. Figure 2, to the same scale, has GSM $B_{x}, B_{y}$, solar wind proton density, and

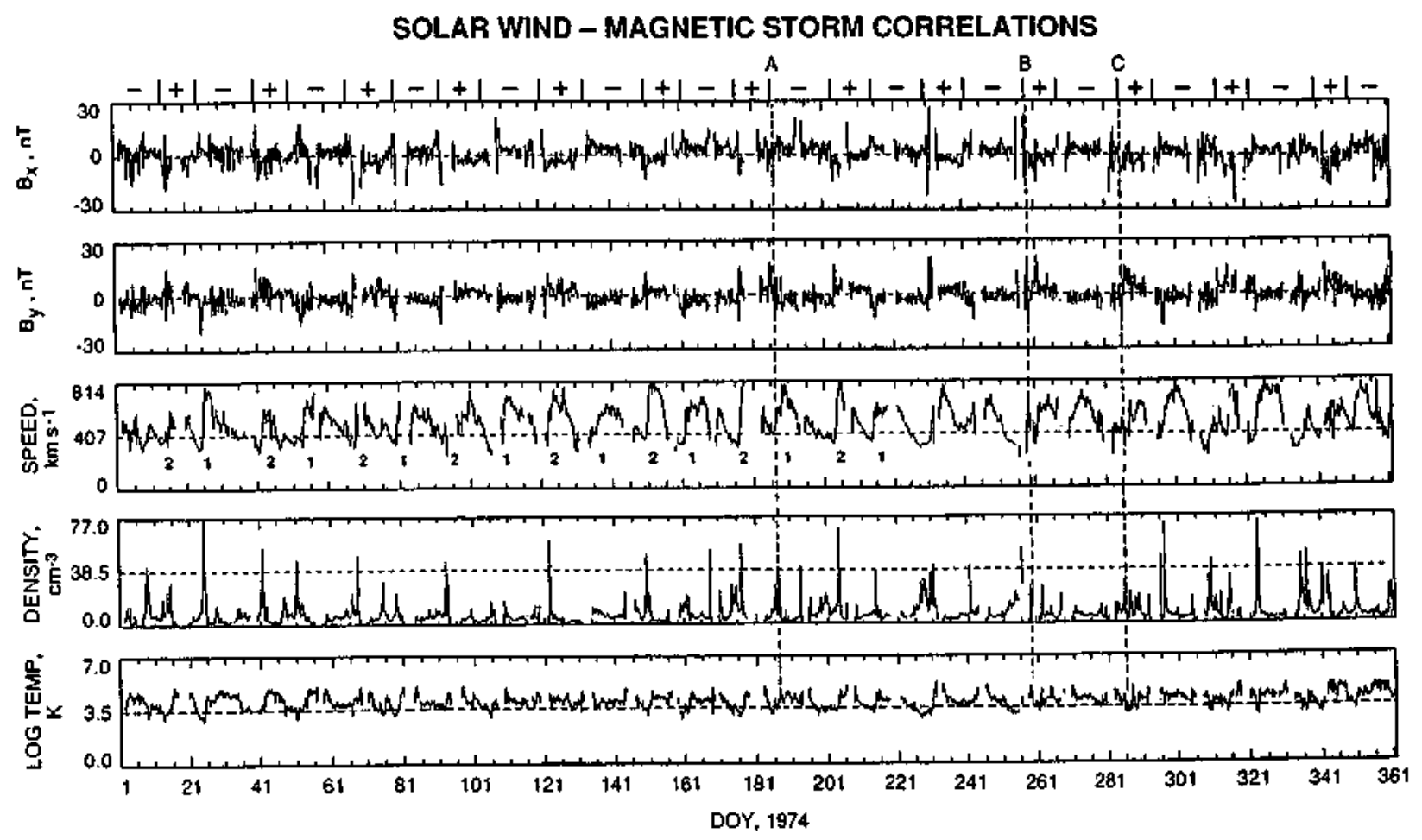

Figure 2. Same format as Figure 1, but for IMF $B_{x}, B_{y}, V_{s w}, N$ and $T$. 
temperature. Solar wind speed is repeated in the middle panel of Figure 2 as a reference, so that this figure can be related to features in Figure 1. At the top of both figures, the IMF sectors are indicated, with the plus and minus signs indicating positive and negative sectors. Positive sectors are those in which the fields are directed outward from the Sun.

There are several important points to note in Figure 1. The first is that there are many high-speed streams present (sometimes more than two per solar rotation) with peak speeds up to $800 \mathrm{~km}$ $\mathrm{s}^{-1}$. Most streams have a characteristic profile of a fast tise in speed and a long decay [Neugebauer and Snyder, 1966]. Some excellent examples of this are the three streams within days 24-68 and the three streams within days $177-214$. Other streams have profiles which indicate that the streams are composed of several partially overlapping events. Examples of the latter can be found within days 161-176 (with "small-amplitude" stream onsets on days $162,163,166$, and 171 ) and within days $25-41$ (with onsets on days 25,28 , and 30 ).

In Figure 1, two main recurring streams are present in the first two thirds of the year. Stream onsets are present for the consecutive 27-day periods of days $24,51,78,105,132,159,186$, 213 , and 240. This sequence also extends back in time into 1973 and also forward into 1975 , but with less regularity than the events discussed above. We will refer to this recurring stream as the sequence 1 stream. A second sequence of streams is located between the sequence 1 streams, with onsets on days $15,41,68$, $95,122,150,177,204$, and 231 . This second recurring stream will be called the sequence 2 stream. None of these streams had fast forward shocks, in agreement with the Smith and Wolf [1976] empirical model. Beyond day 231 the streams become somewhat more complex, so we will focus on the first two thirds of the year.

The bottom panel of Figure 1 shows the hourly averages of the magnetic field magnitude. The highest field magnitudes that occurred during the year are located near the leading edges of the high-speed streams. Almost all of the large fields that have hourly averages greater than $15 \mathrm{n} T$ are of this type. Examples are present on days $25.26,150-151,161-162,176-177,187$, and 204. The maximum (hourly average) intensities for these events lie in the range of 10 to $25 \mathrm{nT}$.

There were only four cases where hourly average field strengths exceeded $30 \mathrm{nT}$. These events occurred on days 187 , 232,258 , and 286 . Three of these events are associated with major (Dst $\leq-100 \mathrm{nT}$ ) magnetic storms, labeled the A, B, and C events. Each is associated with a solar ejecta event and not with a corotating stream. This will be discussed in depth later.

\section{Interplanetary Events Causing Magnetic Storms (Dst)}

The second panel of Figure 1 is the hourly average Dst values for the year. There are two notable features in this panel. The first is that there are only a few major ( $D_{s t} \leq-100 \mathrm{nT}$ ) magnetic storms during the entire year. These are the previously mentioned event $\mathrm{A}$, on day $187(D s t=-205 \mathrm{nT})$, event $\mathrm{B}$ on day 257 (Dst $=$ $-140 \mathrm{nT})$, and event $C$ on day $285(D s t=-115 \mathrm{nT})$. All three of the high-speed stream events are led by fast forward shocks (shown later). These streams do not recur at 27-day intervals (it is noted however, that the latter two events are separated by 28 days).

The next highest level of magnetic storm intensities during 1974 occurs in a narrow range of $-85 \mathrm{nT} \leq D s t \leq-70 \mathrm{nT}$. There are four storms in this grouping, and they occur on days 75,80 , 108 , and 204. Each of the events is associated with large, $\sim 15-n T$ (hourly average) interplanetary magnetic field magnitudes. These large fields are located in and near the high- velocity/low-velocity stream interfaces. The stream events on days 80 and 108 are sequence 1 corotating streams. The stream event on day 204 is a sequence 2 stream. The event on day 75 is a smaller, impulsive stream located between the two main sequence corotating streams.

The storm intensities associated with the nicely periodic (27day) sequence 1 and 2 stream structures are only modest at best. As noted above, from day 1 to day 241, during nine solar rotations and 18 major corotating stream appearances, there were only three stream appearances associated with $D s t \leq-70 \mathrm{nT}$ values. Most (12 of 18 events) of the above high-speed streams cause only small Dst disturbances of Dst $\geq-50 \mathrm{nT}$. Sometimes the geomagnetic disturbances were not even perceptible in Dst. Examples of this latter type of small ( $\geq-25 \mathrm{nT}$ ) disturbance, or no storm at all are present on days $15,41,68,95,123,134,150,162$, 177,231 , and 241 . The peak speed gradient and peak magnetic fields of these streams are not much different from those that cause storms with intensilies of $D s t \leq-70 \mathrm{nT}$, however.

The top panel of Figure 1 is the IMF $B_{2}$ component in GSM coordinates. Large, southward $B_{z}$ spikes are coincident with storms with $-85 \mathrm{nT} \leq D s t \leq-70 \mathrm{nT}$. Examples can be found on days $74-75,80,108$, and 204 . Other large southward $B_{z}$ spikes (< $.10 \mathrm{nT}$ ) can be noted on days $24-25,75$ and 214 . These events are related to moderate intensity storms. Presumably, magnetic reconnection is the mechanism of solar wind energy input into the magnetosphere for these events.

\section{Geoffectiveness of the Two Corotating Steam Sequences}

The two streams, sequences 1 and 2 , vary in their geoeffectiveness from solar rotation to rotation and over their stream lifetimes. Of the two sequences, the first is more geoeffective at the beginning of the interval studied. Sequence 1 , starting on day 25 and progressing at 27 -day intervals, caused peak Dst values of $-65,-20,-75,-85,-45,-30$, "A," -60 , and $.35 \mathrm{nT}$. The seventh event of the sequence near event $A$ is indeterminate because the large "A" storm overshadows any activity associated with the corotating stream. From Figure 1 the reader can note that the speed of the stream decreases steadily throughout the sequence. The peak speed starts near $\sim 800 \mathrm{~km}$ $\mathrm{s}^{-1}$ and declines to $\sim 600 \mathrm{~km} \mathrm{~s}^{-1}$ near the end of the sequence.

Sequence 2, starting at day 15 (and progressing at 27-day intervals) caused peak Dst values of $-20,-20,-30,-50,-30,-25$, $-40,-75$, and $-35 \mathrm{nT}$. The peak speeds of the streams at the beginning of the sequence have values of $-600-650 \mathrm{~km} \mathrm{~s}^{-1}$. This value rises more or less continuously throughout the sequence until a peak speed of $800-850 \mathrm{~km} \mathrm{~s}^{-1}$ is reached for the last four appearances of the stream. A sharp magnetic spike of magnilude 15-20 nT is present at the high-speed stream-slow stream interaction regions during the last appearances of this sequence. Again, there is not a direct relationship between peak solar wind speed and Dst .

It shouid be noted that previous studies have described the general relationship between solar wind speed and geomagnetic activity during this phase of the solar cycle. Sheeley et al. [1976, 1977] and Gosling et al. [1976] have examined the same interval of time (and other intervals as well) and have come up with similar but more general conclusions.

\section{Positive $D_{s t}$ Intervals}

Two other features in the Dst plot are noteworthy. One is that there are several intervals where $D s t$ has positive $(-+10$ to +20 nT) values for several days at a time. These features can be 


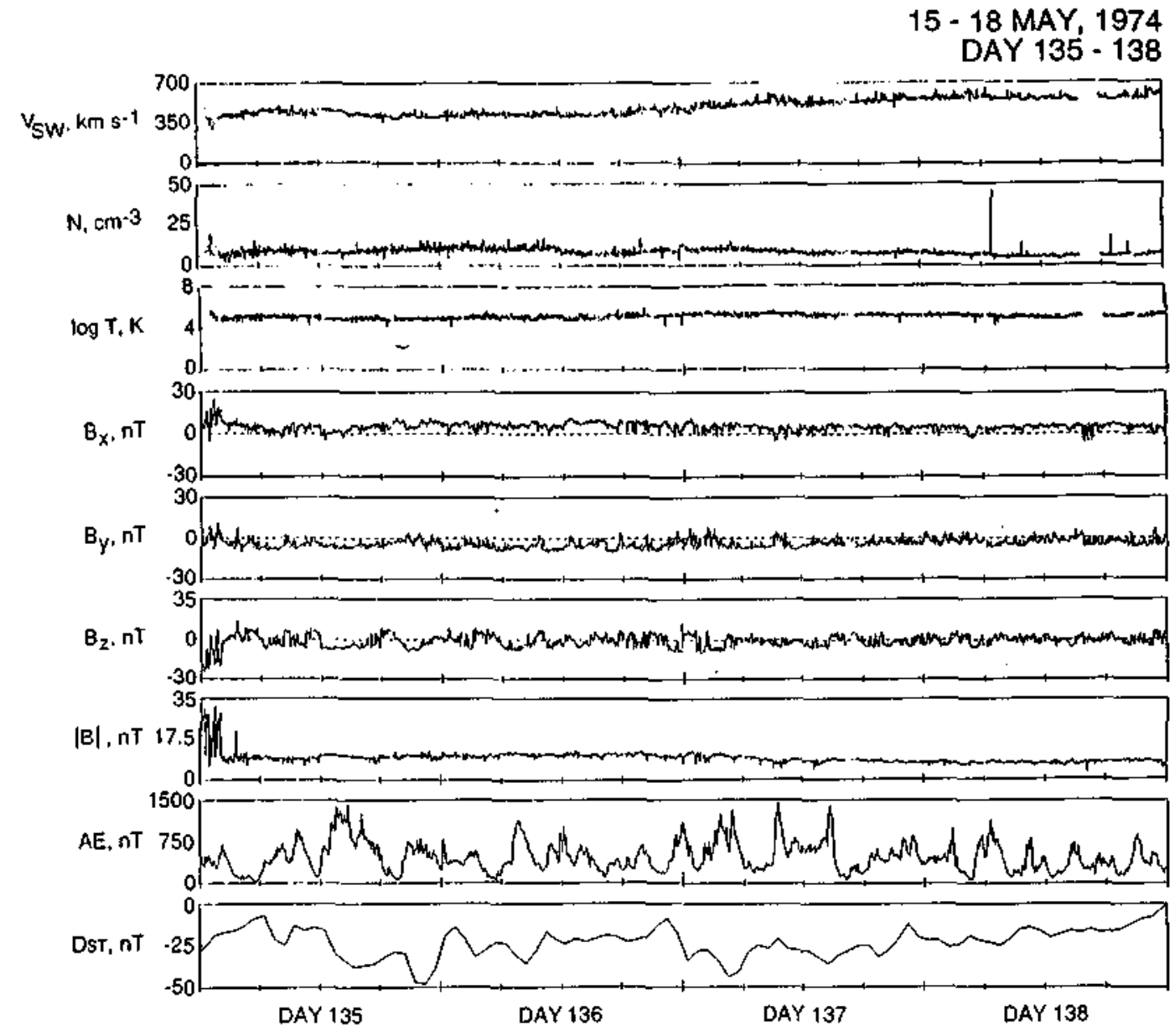

Figure 3. Dst in a "recovery" phase of a magnetic storm. $V_{s w}, N, T$, the IMF, $A E$ and $D s t$ or days 135 through 138, 1974. The ring current does not have a classic decay profile, but $D s t$ stays within the range of $\sim$ $-1010-50 \mathrm{nT}$ for days. Short $D s t$ decreases are accompanied by southward $B_{z}\left(B_{s}\right)$ events and $A E$ increases. Presumably, magnetic reconnection through the $B_{z}$ southward turnings causes the injection of fresh plasma into the magnetosphere, leading to small enhancements of the ring current.

related to specific solar wind parameters. Examples of positive Dst events ate found on days $14,40,50,78,105,132$, and 227. The onsets of these events are gradual, and the magnitude of Dst typically increases with time. The events start days before the appearance of the high-speed streams. Each event is associated with a slow solar wind stream located between the two high-speed coronal hole streams. For the events mentioned above, the minimum solar wind speed was approximately $350,310,330,330$, 310,330 , and $300 \mathrm{~km} \mathrm{~s}^{-1}$, respectively. The densities during these intervals are $20,10,7,12,10,8$, and $30 \mathrm{~cm}^{-3}$, which are either typical of solar wind densities or slightly on the high side. These values increase with time to $20,44,38,20,30,12$, and $40 \mathrm{~cm}^{-3}$. Although the solar wind speeds are low, the high plasma densities cause an increased ram pressure and thus the positive $D_{S t}$ values.

\section{Dst Recovery}

Another feature to note in the Dst panel of Figure 1 is the very long recovery phases for the small magnetic storms generated by the corotating high-speed streams. Most recoveries last 1 week or longer, sometimes even until the next stream arrives. This is substantially different from what happens with storms that occur at and near solar maximum (a standard decay time of the solar maximum storms is $<10$ hours [Gonzalez et al., 1989]. In this epoch, long recovery phases are the rule rather than the exception. Some prime examples of this are found on days 25 to 35,80 to 92 , and 108 to 122 for the sequence 1 streams and days 204 to 214 and 231 to 241 for the sequence 2 streams. Many other examples are present in the figure and for those in 1973 and 1975 (not shown).

Some storm "recoveries" have Dst negative intensities which are relatively constant for days on end. Two good examples of this feature are present on days 52-60 and 135-138. Both of these events are associated with sequence 1 strearns. On day 52 the $D s t$ event onset coincides with a very small amplitude precursor solar wind stream prior to the main, high-speed stream, and the Dst depression continues well into and beyond the speed peak.

The days 135-138 event is shown in higher temporal resolution in Figure 3. The panels are the solar wind plasma parameters, plus interplanetary magnetic fjeld components and magnitude, and $A E$ and $D$ st. There is a moderate speed precursor stream on day 134 (Figure 1) prior to the sequence 1 stream. In Figure 3 the slow rise in speed of the sequence 1 stream starts near the end of day 135 and continues throughout day 138. Dst lies between -10 $\mathrm{nT}$ and $-50 \mathrm{nT}$ for these four days. This region of more or less continuous low-level $D s t$ occurs both on the trailing edge of the precursor stream and on the leading edge of the larger sequence 1 corotating stream. 

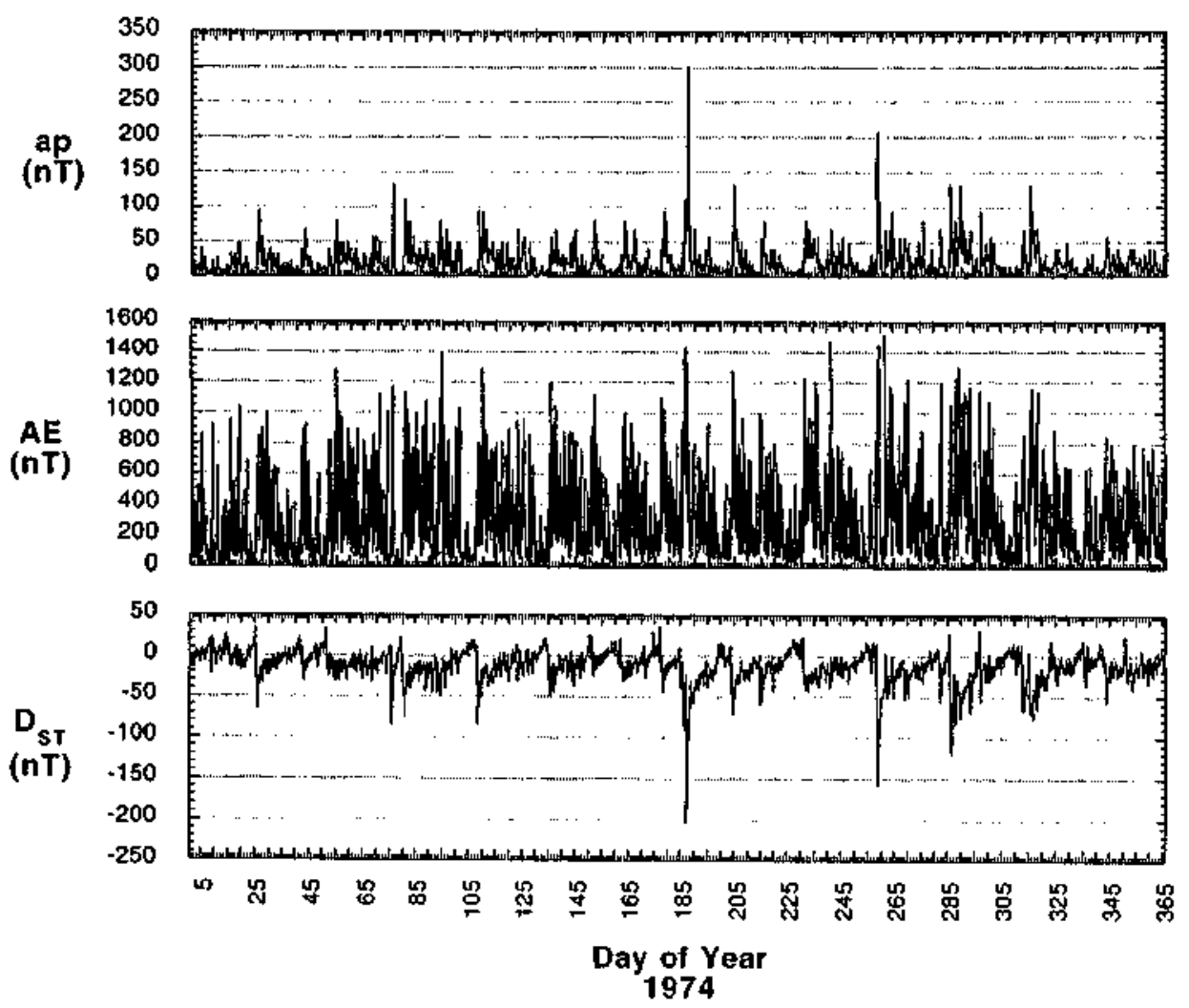

Figure 4. Hourly averages of $a p, A E$ and $D s t$ for 1974.

The causes of these small Dst decreases can be understood by examining simultaneous $A E$ indices. There is almost a one-to-one correspondence between substorms $(A E)$ and ring current increases ( $D s t$ decreases). Substorms are continuously occurring during this 4-day interval. Substorms cause the sporadic Dst decreases and prevent a full recovery of the ring current to quiet day values. This continuous $A E$ activity is a high-intensity Jongduration continuous $A E$ activity (HILDCAA) event (Tsurutani and Gonzalez, 1987]. These substorms are caused by negative $B_{z}$ components which are parts of large-amplitude Alfven waves [Tsurutani et al., 1990]. The Alfvén waves will be discussed later.

All of the other storm long recovery phase events are associated with HILDCAA events. These in turn are associated with large-amplitude Alfvén wave events where the wave components have significant negative $B_{Z}$ components. One possible interpretation of these results is that the $B_{s}$ components of the Alfvén waves lead to magnetic reconnection and substorms, and these substorms lead to the injection of small amounts of particle energy into the outer regions of the magnetosphere where they decay rapidly. More on this topic will be stated in the following section.

\section{Substorm (AE) Relationship to Storms (Dst)}

Figure 4 gives the ap and $A E$ indices for 1974 with the $D s t$ index repeated as a reference. The $A E$ and $D s t$ indices are I-hour values, and the $a p$ indices are 3-hour values. As previously stated, the annual $A E$ average is an exceptionally high value of $283 \mathrm{nT}$ [Saba, 1992]. However, it can be noted that $A E$ rarely attains (hourly average) intensities above $1200 \mathrm{nT}$. All cases of high $A E$ events occur during relatively large magnetic storms. The $A E>1200 \mathrm{nT}$ intervals are found on days $54(\sim 1200$
nT), $94(-1400 \mathrm{nT}), 109(-1300 \mathrm{nT}), 186-187(-1400 \mathrm{nT}), 204$ $(-1200 \mathrm{nT}), 231(-1200 \mathrm{nT}), 241(\sim 1400 \mathrm{nT}), 259(-1400 \mathrm{nT})$, $270(-1200 \mathrm{nT})$, and $289-290(-1300 \mathrm{nT})$. Events on days $186-$ $187,204,231$, and 288.289 occurred during storm main phases. On days $54,109,259$, and 261 the peak $A E$ events occurred during storm recovery phases. For the latter cases, significant ring current intensifications (Dst decreases) are associated with these $A E$ events.

To answer the question, "If the peak $A E$ values are not exceptionally large, then what is it that causes the average $A E$ value for 1974 to be unusually high?", we compare the $A E$ index to $D s t$ for sequence 1 . Starting on day 25 , we note that $A E$ reaches a peak intensity of $\sim 800 \mathrm{nT}$ within one-half day, corresponding quite closely to the $D s t$ decrease in the storm main phase (day 25 is also shown in Figure 7; 2.5-min average $A E$ values are used for this latter figure). However, $A E$ remains quite high for many days afterward, up through day 31 and beyond. Throughout this period, $D s t$ generally recovers, but there are frequent small $\sim 10$ - to 20-nT decreases, indicating small ring current (energy) injections. The next occurrence of the high-speed stream is on day 53. $A E$ oscillates between $\sim 250 \mathrm{nT}$ and $-1000 \mathrm{nT}$ from day 53 through day 60 . The solar wind speed declines from a peak value of $\sim 750$ $\mathrm{km} \mathrm{s}^{-1}$ to $-600 \mathrm{~km} \mathrm{~s}^{-1}$ throughout this interval. Dst has values between -10 and $-20 \mathrm{nT}$ with no indication of decaying further. Again, frequent small $D s t$ decreases are associated with $A E$ increases.

The next passage of this sequence 1 high-speed stream occurs on days 79-80. A peak velocity of $\sim 750 \mathrm{~km} \mathrm{~s}^{-1}$ is reached on day 80 , and the stream speed decays to $-540 \mathrm{~km} \mathrm{~s}^{-1}$ by day 86 . On day 79 there is a sharp increase in $A E$ to peak values of $-1000 \mathrm{nT}$. This occurs concurrently with the storm main phase. $A E$ remains high until day 86 , when there is a sharp but short drop and then a return to high values. 

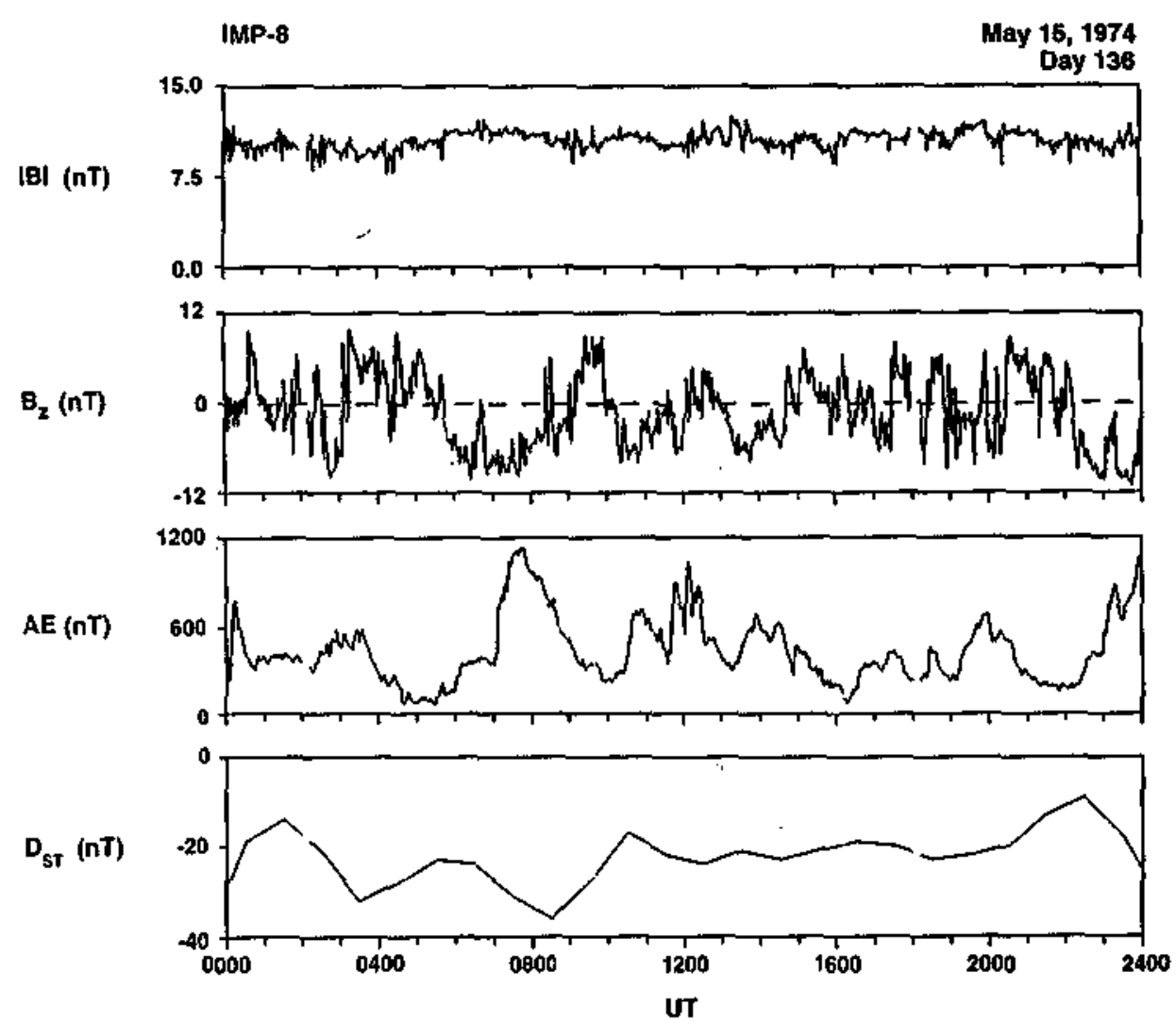

Figure 5. The IMF $B$ magnitude, $B_{z}, A E$ and $D s t$ for day 136, 1974. Increases in $B_{S}$ and $A E$ and slightly delayed increases in $D s t$ are illustrated by this example.

The other appearances of the stream are similar to those discussed above. We skip to the last event where the stream speed and strength have decreased substantially. The eighth appearance of the stream occurs on day 214 . On this day, $A E$ abruptly increases to $-750 \mathrm{nT}$, decreases to $250 \mathrm{nT}$, and increases again to $-800 \mathrm{nT}$ on day 215. Two $D s t$ decreases correspond to these two $A E$ events, $A E$ remains relatively high (100 to $600 \mathrm{nT}$ ) through day 222, after which $A E$ decreases below $200 \mathrm{nT}$. The solar wind speed decreases to $<500 \mathrm{~km} \mathrm{~s}^{-1}$ after day 222 . The association among $V_{\mathrm{sw}}, A E$, and $D_{s t}$ is generally the same for stream sequence 2.

\section{Interplanetary Alfvén Waves and HILDCAAs}

What are the interplanetary feature(s) causing this $A E$ activity? Figure 5 gives an example of the IMF $B_{z}$ component in substantially higher time resolution. Also included in the figure are 2.5-min $A E$ averages, $B$ magnitude, and $D s t$. It can be noted from the $B_{z}$ component of the field that the interplanetary magnetic field is highly turbulent. Some of these field fluctuations have large southward $\left(B_{s}\right)$ values. A one-to-one correlation can be noted between $B_{z}$ decreases and $A E$ increases, indicating that magnetic reconnection is driving magnetospheric substorms on Earth. Because the $B_{\mathrm{s}}$ events associated with these fluctuations are not as intense and do not last as long as those associated with the storm main phase events, particularly for those of the A, B, and C events (here typically $B_{S}-5$ to $-10 \mathrm{nT}$ with durations of $-30 \mathrm{~min}$ to -2 hours), the ring current particle injections caused by the Alfven wave $B_{S}$ events are not as substantial. Other periods have been examined with the same general findings.
A cross-correlation analysis of 24 hours of the plasma and magnetic field $y$ components is given in Figure 6 . At zero lag, $V_{y}$ and $B_{y}$ have a peak correlation coefficient of 0.65 , indicating that these fluctuations are Alfven waves propagating outward from the Sun [Belcher and Davis, 1971; Tsurutani et al., 1990]. The southward deviations in the field associated with these waves are causing the high-latitude (auroral) geomagnetic activity through magnetic reconnection. Other 24-hour intervals where largeamplitude $B_{z}$ fluctuations were present were analyzed. We obtain

$$
a_{Y}-v_{y} \text { cross Corretation }
$$

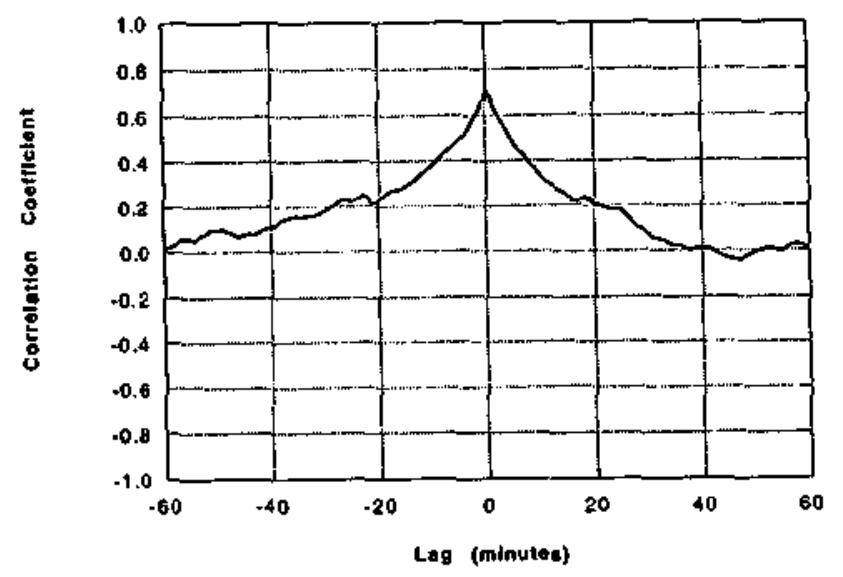

Figure 6. Cross correlation between the solar wind $V_{y}$ and $B_{y}$ for day 136, 1974. The peak correlation coefficient at zero lag indicates that the $B_{y}$ and $v_{y}$ fluctuations are Alfven waves propagating outward from the Sun. 
similar correlation coefficients peaked at zero lag. If the time intervals of analyses are shortened to 12 or 6 hours, the correlation coefficient increases to 0.8 or larger.

These relatively intense $A E$ events presented here are similar to the HILDCAA events previously noted during solar maximum [Tsurutani and Gonzalez, 1987; Tsurutani et al., 1990]. The only apparent difference is that the $A E$ events discussed here do not necessarily fit the stringent requirements that the $A E$ index remain above the 200-nT threshold for 48 hours and that $A E<200-n T$ intervals are less than 2 hours in duration. This somewhat arbitrary HILDCAA definition was/is artificial, and the important thing here is that more or less continuous auororal substorms (HILDCAAs) are produced by the $B_{s}$ components of interplanetary Alfvén waves.

The cause of the strong $A E$ activity during 1974 is the presence of the two high-speed corotating streams that contain largeamplitude Alfven waves throughout the streams. The waves are almost continuously present, far more so than during solar maximum intervals. When this plasma and field impinge on the Earth's magnetosphere, the southward field turnings associated with the wave fluctuations cause magnetic reconnection and consequential high levels of $A E$ activity. The waves appear to be most geoeffective when the solar wind speeds are near their maximum values. $A E$ is found to decrease with decreasing solar wind speed, particularly when $V_{S W}$ decreases below $500 \mathrm{~km} \mathrm{~s}^{-1}$. Speed is probably not the only physical cause, however. The wave amplitudes are larger in the region of peak speed and generally decrease with decreasing speed.

\section{Twenty-Seven-Day Periodic Quiet Geomagnetic Intervals}

Intervals of geomagnetic quiet have been noted as well (see Figure 1). There are small intervals where $A E$ is generally less than $200 \mathrm{nT}$ for days at a time. These events are found in intervals that precede the onset of the high-speed corotating streams. Examples of events somewhat prior to the sequence 1 streams are found on days 22-24, 49-50, 77-78, 102-105, 130-133, and 224-227. Each of these intervals also partially overlaps with intervals where $D s t$ is positive (discussed previously).

Four of the six low- $A E$ events mentioned above have common interplanetary features. In these cases the velocities decrease from 560 to $310 \mathrm{~km} \mathrm{~s}^{-1}, 520$ to 300,460 to 360 , and 400 to 360 (with a minimum of $300 \mathrm{~km} \mathrm{~s}^{-1}$ ), respectively. The magnetic field magnitude ranges from 6 to $3 \mathrm{nT}, 7$ to 5,6 to 8 , and 5 to $3 \mathrm{nT}$ during these intervals. The $B_{z}$ component variability during these intervals was generally small. There is a general absence of Alfvén waves. There also may be a slight tendency for these intervals to have an average positive $B_{z}$ value.

Several plasma features are generally consistent during the above four events. They occur within the far trailing portion of high-speed streams. The speeds start near $500 \mathrm{~km} \mathrm{~s}^{-1}$ and decrease with time. The temperatures also decrease and the plasma densities increase throughout the events. The densities start at values near the lowest detected in solar wind, $-3 \mathrm{~cm}^{-3}$, and increase to substantial values (as the HCS is approached). For the five cases, $N$ increases from 23 to $65 \mathrm{~cm}^{-3}$ (just prior to the stream onset and HCS crossing at the start of day 25), 3 to $12 \mathrm{~cm}^{-3}$ (including a broad HCS crossing from days 105-106), 3 to $11 \mathrm{~cm}^{-3}$ (just prior to a HCS crossing at the end of day 133), and 5 to 40 $\mathrm{cm}^{-3}$ (just prior to the HCS crossing at the beginning of day 231). The higher ram pressures associated with the enhanced interplanetary densities and the lack of ring current energy injections (due to the presence of relatively weak $|B|$ without directional fluctuations) are the causes of the positive $D s t$ values.

The two events that did not follow this general pattern are found on days $49-50$ and 77-78. The first occurred in a low solar wind speed region, 340 to $430 \mathrm{~km} \mathrm{~s}^{-1}$, just prior to the HCS crossing on day 51 . The density was relatively high, 10 to 30 $\mathrm{cm}^{-3}$. The magnetic field varied from 9 to $4 \mathrm{nT}$. All of these plasma and field features are similar to those occurring near the end of the previous five events. The only difference is that the event was not associated with a trailing portion of a high-speed stream. The magnetic field was also distinctly northward during this interval.

The final quiet event of the seven events, the event on days 77 78 , is associated with a mocierate speed impulsive stream which is located midway between a sequence 2 stream (onset on day 68) and a sequence 1 stream (onset at the end of day 79). The small stream creates a large magnetic magnitude compression on days 75-76 and a magnetic storm onset on day 75 associated with an intense $B_{s}$ event at the front end of the stream. In the quiet interval the IMF magnitude decreases from $15 \mathrm{nT}$ to $5 \mathrm{nT}$, and the density varies from 15 to $4 \mathrm{~cm}^{-3}$. The HCS crossing occurs at the beginning of day 79 , just after the geomagnetic quiet event. The main cause of this particular interval of geomagnetic quiet is that $B_{z}$ is positive throughout the stream interval.

\section{Twenty-Seven-Day Recurrence in ap Activity}

The top panel in Figure 4 is the ap index. This index is constructed from midiatitude magnetometer stations. The index is sensitive to and therefore represents both ring current and auroral activity. By using ap alone, it is difficult/impossible to tell whether the activity is storm (Dst) activity or high-latitude substorm $(A E)$ activity. The $a p$ index is largest during the main phases of the largest storms. However, the index also picks up both the sequence 1 and sequence 2 corotating stream Alfven wave-related events as well. There is a clear 27 -day periodicity in this index. The 1973 and 1975 data contain similar features. The most intense $a p$ intervals are those associated with impulsive streams (ejecta events), but there are clear 27 -day periodicities associated with the coronal hole-associated streams as well. Thus if one examined $a p$ indices alone, one could obtain the impression that there were intense 27-day recurring geomagnetic storms rather than 27-day recurring intense high-latitude auroral aclivity (there are recurring magnetic storms but they are low in intensity). From these 3 years of study there are no 27-day recurring intense (Dst $\leq-100 \mathrm{nT}$ ) magnetic storns.

\section{Corotating Stream Case Studies}

To illustrate the types of phenomena associated with southward $B_{z}$ values that lead to the moderate $D s t$ magnetic storms during 1974, we show two specific interplanetary corotating storm examples. We will examine our high-speed corotating stream events at the beginning of the sequence 1 streams and an event near the end of the sequence 2 streams.

\section{Day 25}

Prior to the beginning of the event illustrated (Figure 7), the field has exceptionally low values of $\sim 1 \mathrm{nT}$ at 1445.1700 UT on day 24 and has an orientation that is in neither a plus or minus sector polarity (see Figures 1 and 2 ). The solar wind has a low 


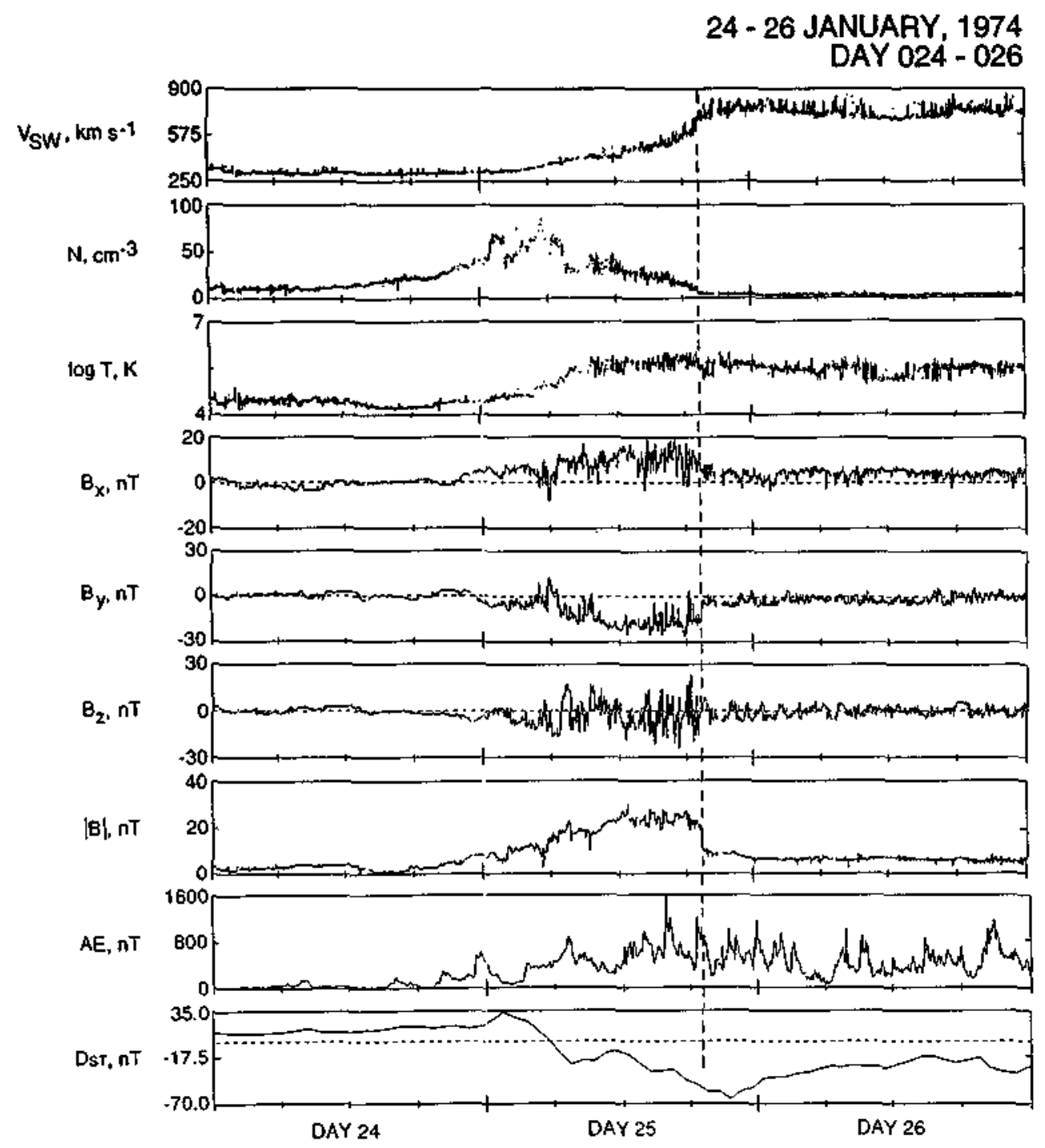

Figure 7. The $D_{s t} \sim-65 \mathrm{nT}$ storm created by a corotating stream/heliospheric current sheet (HCS) interaction on day $25,1974$.

velocity (300-310 $\left.\mathrm{km} \mathrm{s}^{-1}\right)$ as well as a low proton temperature $(-2$ $\left.\times 10^{4} \mathrm{~K}\right)$. The plasma density is high, $-20.30 \mathrm{~cm}^{-3}$, and causes positive $D_{\text {st }}$ values. These plasma features give a good indication that the spacecraft (and Earth) is probably near the HCS [see Winterhatter et al., 1994]. The low magnetic field strengths (and therefore low potential $B_{2}$ values) cause very low $A E$ values, as discussed previousiy.

From 1700 UT on day 24 to 1200 UT on day 25 there are steady trends in several of the plasma parameters. The magnetic field increases from $\sim \mathrm{nT}$ to $25 \mathrm{nT}$. $N$ increases from $15 \mathrm{~cm}^{-3}$ to $80 \mathrm{~cm}^{-3}$ (at -0500 UT) and then decreases to $30 \mathrm{~cm}^{-3}$. There are no significant velocity and temperature gradients until -0400 UT on day 25. At the beginning of this interval the solar wind speed starts at $330 \mathrm{~km} \mathrm{~s}^{-1}$ and rises, and the temperature starts at $3 \times 10^{4}$ $\mathrm{K}$ and rises. By $1200 \mathrm{UT}$, day $25, V_{s w}$ is $\sim 450 \mathrm{~km} \mathrm{~s}^{-1}$ and $T$ is $-5.5 \times 10^{5} \mathrm{~K}$. The increasing plasma density leads to an increase in the solar wind ram pressure and hence a peak Dst of $\sim+35 \mathrm{nT}$ at -0200 UT on day 25 .

Beyond 1200 UT on day 25 the velocity continues to increase, reaching a maximum of $775 \mathrm{~km} \mathrm{~s}^{-1}$ at $\sim 1900 \mathrm{UT}$ on day 25 . The temperature reaches a maximum of $-6 \times 10^{5} \mathrm{~K}$ from 0800 to 1910
UT on day 25 and suddenly decreases to $4 \times 10^{5} \mathrm{~K}$ at $1910 \mathrm{UT}$. This abrupt decrease is due to the presence of a reverse shock, identified in the plasma and field parameters. The solar wind speed jumps from $620 \mathrm{~km} \mathrm{~s}^{-1}$ to $740 \mathrm{~km} \mathrm{~s}^{-1}$, the density abruptly decreases from 12 to $5 \mathrm{~cm}^{-3}$, and the magnetic field magnitude decreases from 22 to $10 \mathrm{nT}$. This example of a reverse shock occurring at $1 \mathrm{AU}$ is fairly common $(-20 \%)$ in the corotating streams studied. Note that the decrease in field magnitude is also accompanied by a decrease in $\boldsymbol{B}_{s}$. The reverse shock causes a sharp decrease in $B_{s}$ (in time) and starts the recovery phase of the storm. This is the opposite of the (forward) shock compression events causing storm onsets [Tsurutani et al, 1988, and events A and $B$ of this paper].

Although there is a clear reverse shock, there is no accompanying forward shock. This is contrary to the general picture presented by Smith and Wolf [1976], which has forward shocks occurring at $-1.5 \mathrm{AU}$ and reverse shocks at $2.5 \mathrm{AU}$. One speculation for the lack of a companion forward shock is the unusual plasma conditions upstream of the corotating stream (the HCS and its plasma sheet). The density is very high (up to 80 $\mathrm{cm}^{-3}$ ) at this interaction region, and the magnetic field strength $|B|$ 
$-25 \mathrm{nT}$ is intense because of the stream-stream interaction. After 1300 UT on day 25 , when the velocity gradient is steepest, the density is $20-25 \mathrm{~cm}^{-3}$ and decreasing. The field is a relatively constant $25 \mathrm{nT}$. Thus the local Alfven speed is $90 \mathrm{~km} \mathrm{~s}^{-1}$ at 1300 UT and increases with time. The solar wind speed is only $480 \mathrm{~km}$ $\mathrm{s}^{-1}$, so the lack of a stronger velocity gradient and the relatively high Alfvén wave speed may prevent a forward shock from forming. See also discussion by Belcher and Davis [1971], Hundhausen [1973, 1985] and Pizzo [1985] for concepts/models of fast shock formation in the heliosphere.

The location of the HCS is somewhat difficult to place in this case. The IMF is clearly a negative sector polarity (positive $B_{x}$. negative $B_{y}$ ) after $\sim 0000$ UT on day 25 . Prior to that time the polarity is mixed or is in an orthospiral (orthogonal to the normal Parker spiral) orientation. The largest plasma densities are on the same side of the HCS as the high-speed stream, possibly indicating that this may be compression due to the stream-stream interaction. However, it should be noted that the magnetic field intensification is found well behind the density enhancement.

The $B_{z}$ that causes the storm is clearly related to the corotating stream interaction with the HCS and its plasma sheet. $B_{z}$ steadily decreases from $+3 \mathrm{nT}$ at 1200 UT on day 24 to $-10 \mathrm{nT}$ at 0400 UT on day 25 as the HCS plasma sheet density increases from 12 to $60 \mathrm{~cm}^{-3}$. The most intense $B_{s}$ field during the event occurs from 0600 UT to 0655 UT on day 25 with a peak intensity of $16 \mathrm{nT}$. This intense southward IMF is coincident with the magnetic storm onset. The location of this $B_{s}$ event is found at the base of the HCS plasma sheet/stream interface. The speed of the stream is only $370 \mathrm{~km} \mathrm{~s}^{-1}$ at the time.

It is noted that the $B_{s}$ fluctuations causing the storm are parts of large-amplitude $B_{z}$ variations. The origins of the intense $B_{z}$ fluctuations are not presently known. They may be locally generated from stream-stream interaction effects, or they may be amplified Alfven waves from the coronal hole stream. Tests are currently being nun. We note that the field magnitude decrease across the reverse shock (in time) is a factor of $\sim 2.5$. We also note that the Alfvén wave transverse amplitudes decrease by a similar factor. This finding is suggestive that the latter hypothesis may be the correct one.

Recent Ulysses results are in accord with the suggestion that the above fluctuations are Alfven waves. The Ulysses magnetic field and plasma results have indicated that polat coronal holes consist of a relatively steady high-speed stream of $750-800 \mathrm{~km}$ $s^{-1}$ [Phillips et al., 1994] with the presence of large-amplitude $(\Delta \mathbf{B} /|B| \sim 1-2)$ Alfven waves throughout the stream [Tsurutani $e t$ al., 1994; Smith et al., 1995]. Presumably, these are the same solar wind structures that reach the ecliptic plane during the descending phase of the solar cycle. The embedded fluctuations would then propagate/be convected into and become part of the stream-stream interaction region (up to the high-speed stream/low-speed stream interface, assuming it is a tangential discontinuity).

\section{Day 177}

The corotating stream event for days 176 through 179 is illustrated in Figure 8. At 1200 UT on day 176, well prior to the stream onset, $V_{s w}$ is $\sim 315 \mathrm{~km} \mathrm{~s}^{-1}, N$ is $-10 \mathrm{~cm}^{-3}, T$ is $-5 \times 10^{4} \mathrm{~K}$, and $|B|$ is $-6 \mathrm{nT}$. At the very beginning of the stream gradient, $-0000-0600 \mathrm{UT}$ on day 177 , the density increases to $35-65 \mathrm{~cm}^{-3}$. The increased solar wind ram pressure causes $D_{s t}$ to reach $\sim+35$ $\mathrm{nT}$ during this interval. Since the solar wind speed increase is only slight, the $D_{s t}$ (ram pressure) increase is due almost entirely to the density enhancement.

The magnetic field magnitude also abruptly increases with the density increase. Peak values of $-25 \mathrm{nT}$ are reached near $\sim 0600$ UT on day 177. However, somewhat different from the plasma density profile, the magnetic field intensity remains high until $\sim 1500$ UT on day 177. Thus the density enhancement is located at the very base of the corotating stream and the magnetic field enhancement further within the stream gradient. The corotating stream peak speed of $750-800 \mathrm{~km} \mathrm{~s}^{-1}$ is attained at -0000 UT on day 178 when the field has decreased to a steady $\sim 7 \mathrm{nT}$.

The location of the HCS can be noted by examining the IMF $B_{y}$ and $B_{x}$ components. Prior to $-0500-0600$ UT on day $177, B_{x}$ is generally negative, and after that time, $B_{y}$ is positive. There is a broad discontinuity from $\sim 0500$ to 0600 UT, when $B_{y}$ reverses sign. Prior to $\sim 0500$ UT, $B_{x}$ is positive, and after $\sim 1000$ UT, $B_{x}$ is generally negative. Thus one can possibly interpret the -0500 to 0600 UT interval as a crossing from a negative sector field to a positive sector field.

The largest and longest duration negative $B_{z}$ event occurs at this transition. $B_{z}$ has a value of $\sim-15 \mathrm{nT}$ for $\sim 1$ hour. This is coincident with the $B_{y}$ sign reversal. The negative $B_{z}$ event marks the start of the small storm (Dst $\leq-15 \mathrm{nT}$ ). The nature of the field within the high field region is again of a highly fluctuating nature. These fluctuations could be interpreted as amplified Alfvén waves. Subsequent negative $B_{z}$ values on days 178.179 , associated with Alfven waves, lead to a peak $D s t--45 \mathrm{nT}$ on day 178.

\section{HCS Crossings and Geomagnetic Activity}

We have examined all of the other magnetic storm events occurring during 1974 with $D s t \leq-50 \mathrm{nT}$. We wish to determine whether there is any dependence on the polarity of the IMF, a dependence that might be present if the equinoctial hypothesis is the predominant cause of these smaller storms [Russell and McPherron, 1973; Crooker ef al., 1992a), (however, see discussion by Gonzalez et al. (1993]). We have also examined the data for possible storm seasonal dependences. There are fourteen $D_{s t} \leq-50 \mathrm{nT}$ events in all. Seven of these are associated with $\mathrm{HCS}+/$ - crossings and seven are associated with $/ /+$ crossings. Thus there is no obvious HCS crossing polarity dependence. Although there is no HCS crossing dependence, there is some evidence that negative sectors within March-April and positive sectors within September-October tend to favor Dst enhancement, in agreement with the Russell-McPhetron mechanism. This slight effect can be seen in Figure 1.

\section{Major Storm (Dst $\leq-100 \mathrm{nT})$ Case Studies}

\section{Event A}

The "A" event was the largest magnetic storm tha' occurred during 1974. It can be noticed in Figure 1 that the stream(s) responsible for the storm do(es) not occur either 27 days before or after. In the figure it can be noted that the storm is composed of three distinct main phase components, each main phase more intense than the previous one. These main phases are related to three separate streams, each led by fast-forward shocks. Each stream is nonrecurring rather than corotating.

In Figure 9 we show the high-resolution $B_{2},|B|$, speed, density, and temperature values for the A event from 1200 UT on day 185 


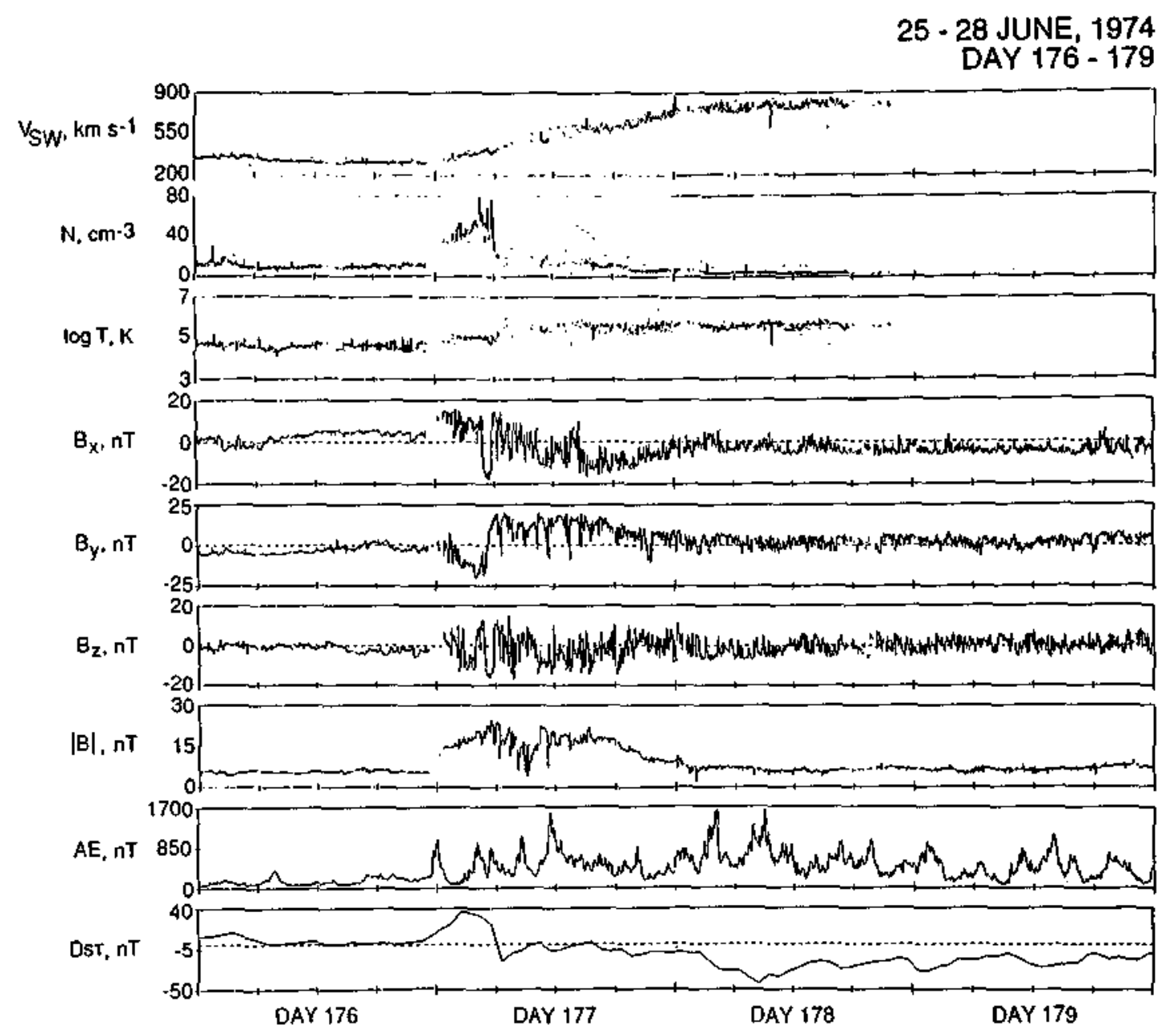

Figure 8. A Dst $-.45 \mathrm{nT}$ magnetic storm created by a corotating stream/HCS plasma sheet interaction on days $177-178$.

until 2400 UT on day 187 . The first fast-forward shock occurs at 0109 UT on day 185 (not shown). The magnetic field compression associated with this shock crossing was relatively small owing to the low stream speed of only $490 \mathrm{~km} \mathrm{~s}^{-1}$. Figure 9 shows the second forward shock at 1532 UT on day 185 (indicated by a dashed vertical line). The peak stream speed is again only $\sim 490 \mathrm{~km} \mathrm{~s}^{-1}$. The magnetic field magnitude increases from $-9 \mathrm{nT}$ to $15 \mathrm{nT}$ across the shock. Well behind the shock at 2245 UT there is a sharp IMF discontinuity in which the field changes from a northward value to one directed southward. The $B_{s}$ component becomes -9 to $12 \mathrm{nT}$ and remains at this value throughout the stream. A peak Dst value of $-85 \mathrm{nT}$ develops at $0700 \mathrm{UT}$, day 186. The most intense main phase onset is relared to the third, higher-speed ejecta. The speed increases from an upstream value of $-450 \mathrm{~km} \mathrm{~s}^{-1}$ to a postshock speed of $600 \mathrm{~km}$ $\mathrm{s}^{-1} . B_{s}$ increases from a value of $10 \mathrm{nT}$ upstream to over $\sim 25 \mathrm{nT}$ downstream. The cause of the intense $B_{s}$ event that causes the storm is shock compression of preexisting upstream $B_{s}$ fields.

From 0410 UT until 0640 UT, day 187, the density is in the range of $-35 \mathrm{~cm}^{-3}$, and the temperature is $\sim 2 \times 10^{5} \mathrm{~K}$ (not shown). The high plasma densities and temperatures do not fit with the possibility of this being part of the driver gas for the event.

\section{Event B}

This interplanetary event is another nonrecurring event led by an interplanetary shock (see Figure I). The event is shown in high resolution in Figure 10. The storm sharp onset is again caused by shock compression of preexisting southward fields. The $B_{z}$ field is $\sim-15 \mathrm{nT}$ prior to the shock (causing a decrease in Dst), becomes compressed to <-40 nT at the shock at $1350 \mathrm{UT}$ on day 258 , and remains at high negative values until 1432 UT. $B_{2}$ then oscillates between $+5 \mathrm{nT}$ and $-40 \mathrm{nT}$ until $1622 \mathrm{UT}$, when it remains at -40 nT. $B_{2}$ then maintains a -40 to -50 -nT level until 1830 UT on day 258.

We have searched for a classical driver gas, using most of the Zwickl et al. [1983] criteria (low temperatures, enhanced He abundances, and quiet magnetic fields), but have not found an obvious region. The fields between 1830 and $2400 \mathrm{UT}$, day 258 , are large and somewhat devoid of waves. The interval has low plasma densities $\left(1-5 \mathrm{~cm}^{-3}\right)$ as well. However, the temperature remains tigh $\left(-6-8 \times 10^{5} \mathrm{~K}\right)$ throughout this region, contrary to the Zwickl et al. criteria for a driver gas. Although it is possible that this may be a driver gas (without all Zwickl et al criteria met), it is not the major cause of the $B_{5}$ event responsible for the storm main phase and does not warrant further study within this paper.

What is the cause of the highly turbulent fields within the above $B_{z}$ event? The answer can be found by looking at the other field components and the field magnitude. Prior to the shock passage, the IMF is in the orthospiral direction with large negative $B_{y}(\sim-10 \mathrm{nT})$ and an even larger negative $B_{z}(-15$ to $-20 \mathrm{nT})$ component. In the interval (1432 to 1622 UT) when $B_{2}$ varies from $+5 n$ T to $-40 n T, B_{x}$ is large and positive (20 to $30 n T$ ), and 


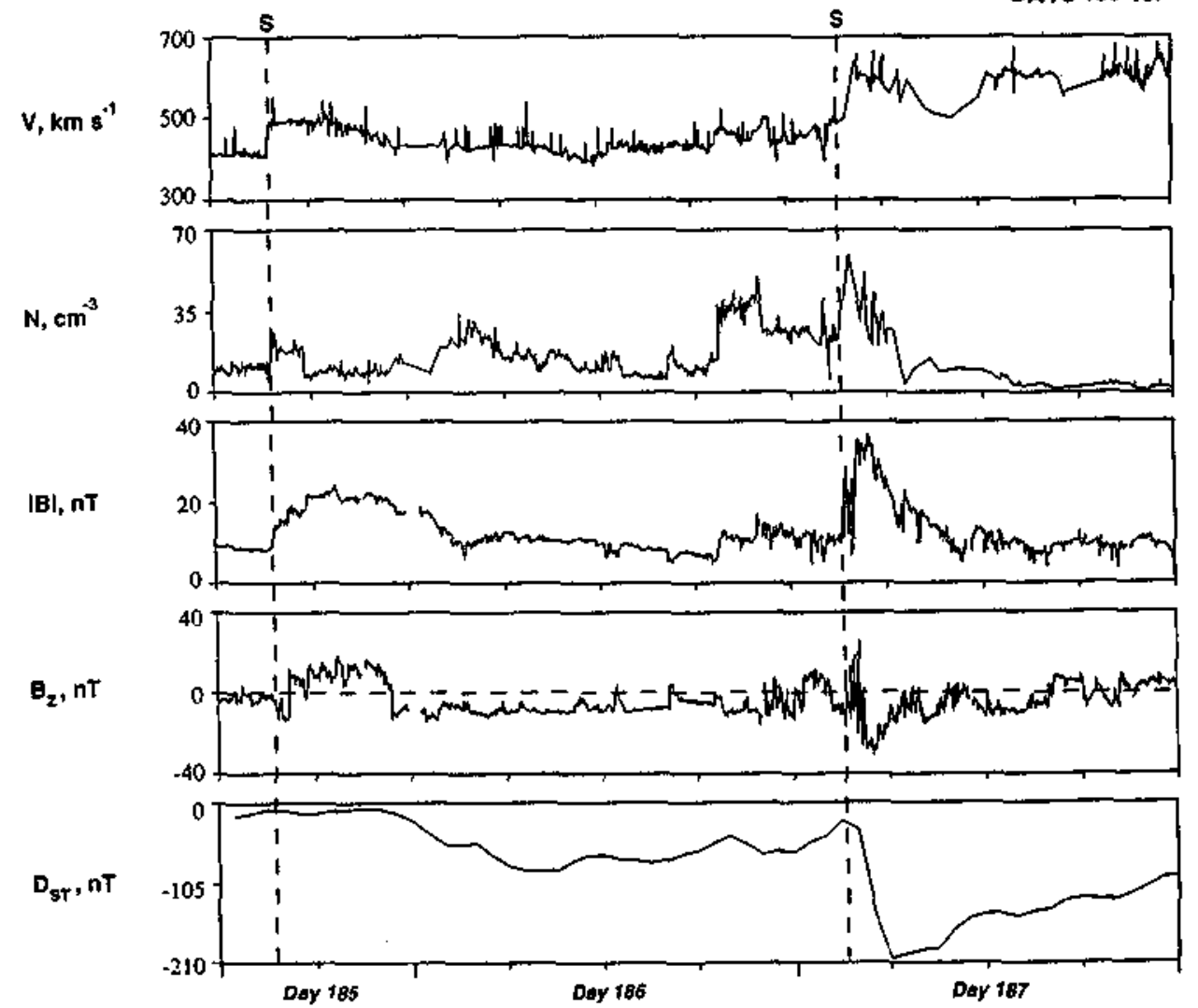

Figure 9. Two of the streams and shocks responsible for the day 185-187 complex magnetic storm (the A event). The solar wind speed, density, magnetic field magnitude, and $B_{z}$ component are displayed. Hourly average $D s t$ is given in the bottom panel.

EVENT B

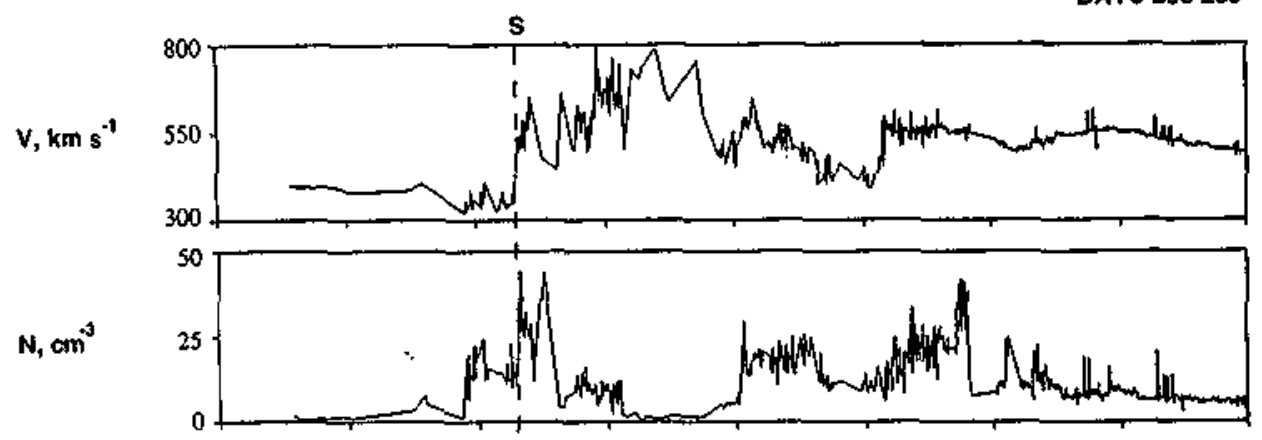

$|B|, n T$

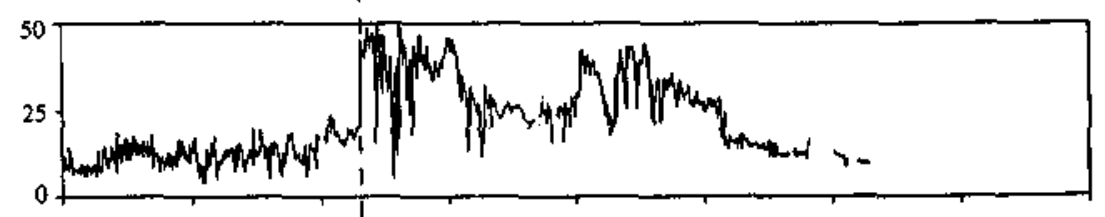

$B_{2}, \mathrm{NT}$
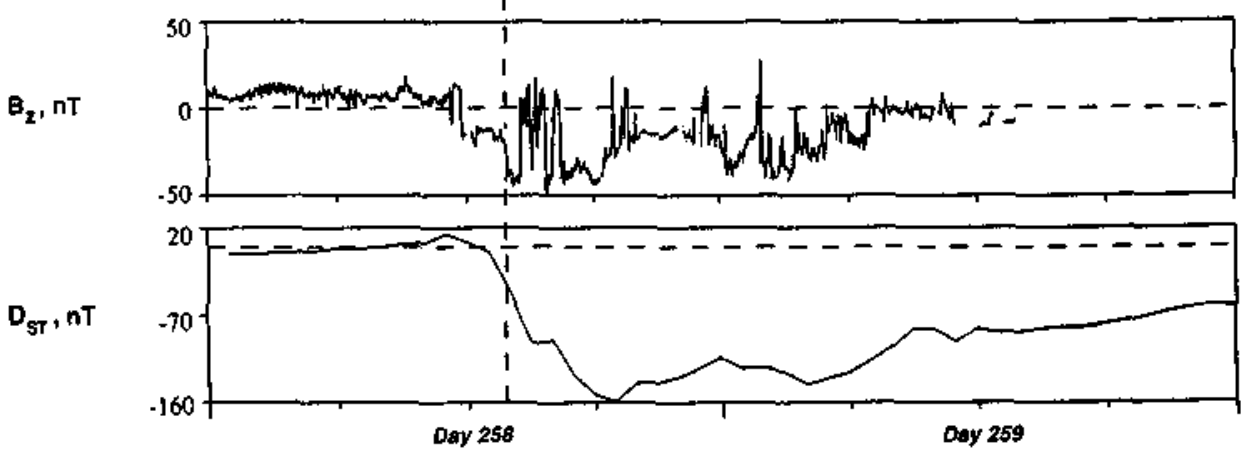

Figure 10. The B storm event. The $B_{s}$ event responsible for the storm is created by shock compression of upstream $B_{s}$ fields. 
$B_{y}$ is positive ( 0 to $10 \mathrm{nT}$ ) but less intense than $B_{\mathrm{X}}$. There are also large magnetic field magnitude decreases associated with the field directional changes, indicative of the presence of strong local currents. After the field flucruation region, from 1622 UT and later, the $B_{x}$ and $B_{y}$ fields are well ordered, with values of $-10 \mathrm{nT}$ and $+10 \mathrm{nT}$, respectively. This is a positive sector field orientation. Thus one possible interpretation of the field fluctuations between 1432 and 1622 UT is that this is a multiple crossing of the heliospheric current sheet or the crossing of several separate current sheets [Crooker et al., 1992b]. Prior to the highspeed event the plasma density is high and the velocity low (not shown), indicating an approach to the HCS region. The clear orientation of the field afterward is perhaps the strongest evidence for the hypothesis of a current sheet crossing.

\section{Event C}

The third most intense storm during 1974 is again associated with a nonrecurring stream led by a fast-forward shock (Figure 11). The shock event occurs at 1245 UT on day 285 , when the magnetic field magnitude increases from $5 \mathrm{nT}$ to $-12 \mathrm{nT}$, the solar wind speed from 400 to $500 \mathrm{~km} \mathrm{~s}^{-1}$, and density from $\sim 10$ to $\sim 20$ $\mathrm{cm}^{-3}$. Shock compression of the interplanetary fields increases $B_{z}$ from $+5 \mathrm{nT}$ to $+12 \mathrm{nT}$. The initial phase of the storm (positive Dst values) starts with the shock passage and associated high solar wind ram pressure.

The southward IMF responsible for the storm is associated with the driver gas fields. This is clearly denoted by the smooth magnetic fields detected at 2100 UT extending to 0135 UT on day
287 (the full extent is not shown). The fields are large and smooth with little wave activity and essentially no discontinuities (of all of the criteria used to identify the driver gas this is one of the best presently available; however, no one measure works $100 \%$ of the time). This is consistent with the standard picture of a magnetic cloud [Burlaga et al, 1987] within a driver gas [Zwickl et al., 1983; Tsurutani et al, 1988; for a discussion of the relationship among magnetic clouds, CMEs and driver gases, see Tsurutani and Gonzalez, 1995a]. The magnetic field $B_{z}$ decreases to $-15 \mathrm{nT}$ at $\sim 0200 \mathrm{UT}$, day 286 , and remains at this large value until 1400 UT on day 286. Near the end of the magnetic cloud at $0130 \mathrm{UT}$ on day 287 the $B_{z}$ component rises to $+5 \mathrm{nT}$.

All three interplanetary events are ejecta events. Event $A$ was caused by three superposed streams. Event B is an ejecta event which occurs where the corotating streams begin to become quite complex (as mentioned previously). Event $C$ is due to a stream whose peak speed is less than $530 \mathrm{~km} \mathrm{~s}^{-1}$. This latter event has a clean profile of a fast rise-slow decay signature and is impulsive in nature.

\section{Solar Sources of the A, B, C Interplanetary Events?}

Possible solar sources for the three high-speed streams (A, B, C events) were sought by examining the Solar-Geophysical Data (Boulder, Colorado) and the World Data Center A for SolarTerrestrial Physics, report UAG-52, 1974. The speeds of the high-speed streams at $1 \mathrm{AU}$ were also used to nule out events that would require unreasonably high or unreasonably low transit speeds.

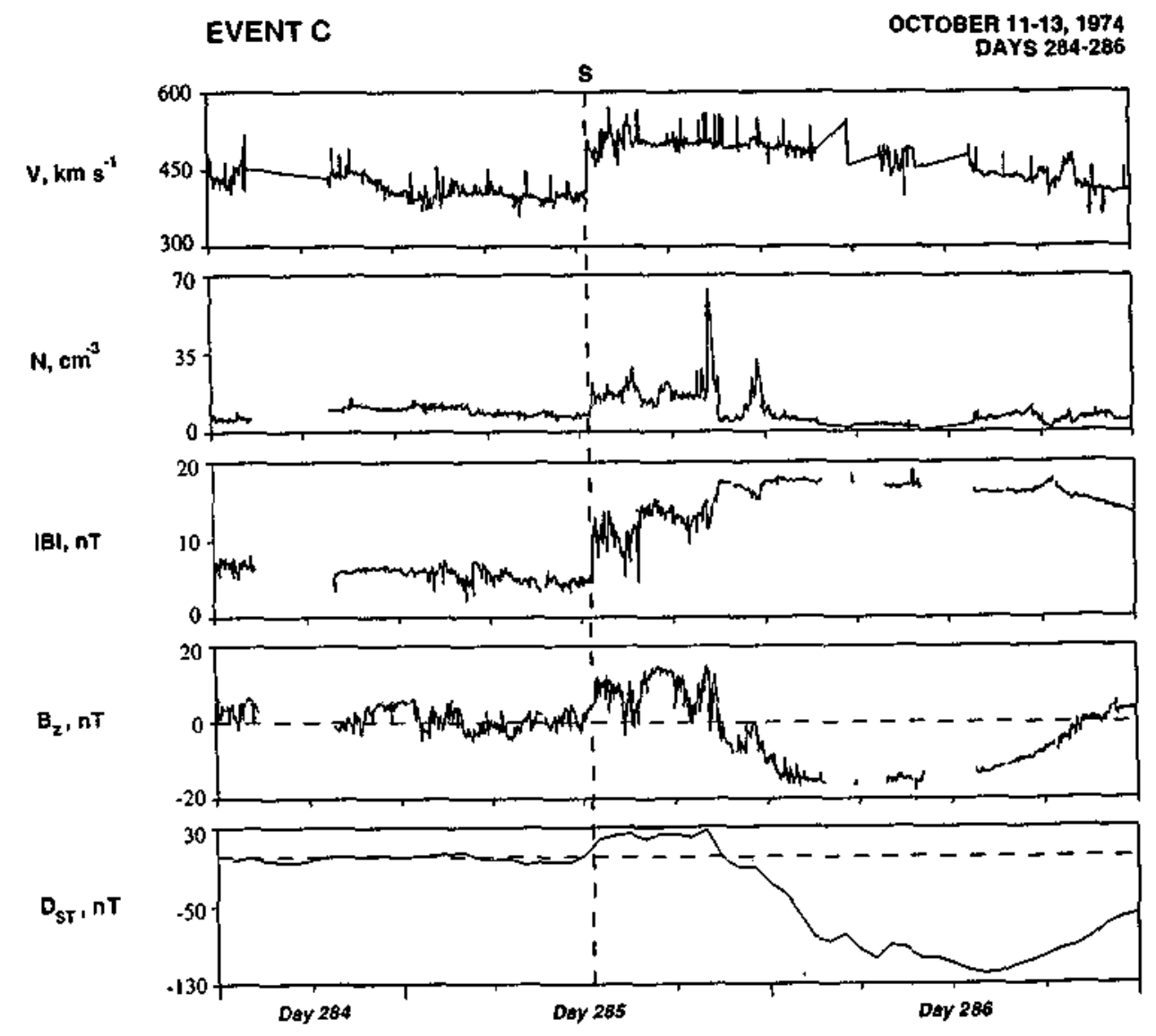

Figure 11. The $\mathrm{C}$ storm event. The storm is created by $B_{S}$ within the driver gas (a magnetic cloud). 


\section{Event A}

Three small 1B/2B flares were the most intense events during this period region (region 13043, day 184, 0259-0318-0412 UT, day 185, 0801-0840-0928 UT, and 1338-1357-1442 UT). Because event $A$ is a compound event involving three interplanetary shocks, one might hope that these three flares would correspond to the three interplanetary shocks at $1 \mathrm{AU}$. However, the timing for the first two flares was within 24 hours of the detection of the shocks at $1 \mathrm{AU}$. Such unreasonably short transit times rule out these flares as possible sources, unless one assumes that very large deceleration is occurring between the sun and $1 \mathrm{AU}$.

The solar flare event at 0259 UT on day 184 is the only event that fits any of the three shocks time-wise. If one considers the last shock occurring at -0000 UT on day 187 , an average speed of $\sim 604 \mathrm{~km} \mathrm{~s}^{+1}$ is determined. This is in good agreement with the measured postshock flow speed of $-600 \mathrm{~km} \mathrm{~s}^{-1}$. However, no solar events could be related to the other two solar wind streams. The latter two are more important in terms of producing the geomagnetic storm. This event can be thought of as a compound stream, as discussed by Burlaga et al. [1987].

\section{Event B}

The interplanetary shock is detected at 1350 UT on day 258 . The postshock flow speed is $\sim 645 \mathrm{~km} \mathrm{~s}^{-1}$. Assuming a constant flow from the Sun to the Earth, this would correspond to a transit time of -2.7 days. The only obvious solar event during this time is a $2 \mathrm{~B}$ flare on September 13. If this solar event is the cause of the interplanetary ejecta, the ejecta would have to have had an average transit speed of $-850 \mathrm{~km} \mathrm{~s}^{-1}$. This is quite high considering the measured speed at $1 \mathrm{AU}$. This solar event seems like an unlikely candidate. There were no prominence disappearance events during the interval of interest.

\section{Event C}

The shock was detected at $1 \mathrm{AU}$ at $1245 \mathrm{UT}$ on day 285 . The only prominent fiare that occurred within the general time interval was on day 284 at $0325 \mathrm{UT}$. There were no prominence disappearance events in the interval of interest. . The 2B flare occurred less than a day and a half prior to the detection of the shock at $1 \mathrm{AU}$. Because the stream speed was quite low, $-500 \mathrm{~km}$ $\mathrm{s}^{-1}$, this solar event seems unlikely to be related to the stormcausing stream.

It has been extremely difficult to identify solar events associated with the $A, B$, and $C$ storm-causing solar wind streams detected at $1 \mathrm{AU}$. These three nonrecurring events probably did not have optical or $\mathrm{X}$ ray counterparts.

\section{Summary and Discussion}

1. In 1974, only moderate ( $\left.-100 \mathrm{nT} \leq D_{s t} \leq-50 \mathrm{nT}\right)$, weak ( $-50 \mathrm{n}$ T $\leq$ Dst $\leq-25 \mathrm{nT})$, or negligible $\left(-25 \mathrm{nT} \leq D_{s t}\right)$ magnetic storms were caused by corotating streams. Corotating streams were not responsible for major (Dst $\leq-100 \mathrm{nT}$ ) magnetic storms. Although the fast stream-slow stream interactions (corotating stream/HCS plasma sheet interactions) created intense fields with hourly average $|B| \sim 15-25 \mathrm{nT}$ and $B_{z} \leq-10 \mathrm{nT}$, the $B_{z}$ component was typically highly fluctuating. The Gonzalez and Tsurutani
[1987] criteria for a major (Dst $\leq-100 \mathrm{nT}$ ) magnetic storm (during solar maximum), a dawn-to-dusk $E_{\text {sw }} \geq 5 \mathrm{mV} / \mathrm{m}$ (approximately comparable to $B_{s} \leq-10 \mathrm{nT}$ ) and $T>3$ hours, were not met at any of the corotating stream compression regions. The $B_{s}$ value was often greater than $10 \mathrm{nT}$, but the time duration was less than 3 hours. Thus it is possible that this empirical criterion derived from a solar maximum interval, may also hold during the descending phase of the solar cycle as well. Similar results were found for 1973 and 1975 as well (not shown).

2. The extremely high annual $2.5-\mathrm{min} A E$ average of $283 \mathrm{nT}$ for 1974 was due to the presence of two corotating streams that contained continuous, large-amplitude Alfven waves. As the magnetic field of the wave rotates southward, magnetic reconnection takes place at the Earth's magnetosphere, and a substorm occurs. As the field rotates northward, it turns off reconnection, and the substorm terminates. The quasi-periodic southward components cause the quasi-periodic substorm activity, called HILDCAAs.

3. The magnetic storms caused by the corotating coronal hole streams have extremely long decay times, sometimes greater than -10 days, even weeks. These are caused by the presence of HILDCAAs within the recovery phase. Substorms comprising the HILDCAAs inject small amounts of ring current particle energy into the outer regions of the magnetosphere that are detected as slight Dst decreases. The continuous substorms (and particle injections) tend to keep the ring current at a quasi-steady state and do not allow it to recover to its quiet time value. The relatively rapid 2- to 3-hour decay of these small Dst events is unexplained at this time, however. One possible explanation is that the particles gradient drift to the magnetopause and are convected into the magnetosheath. Another possibility is the IMF $B_{N}$ part of the Alfven wave may play a role in outward radial convection of the nightside ring current. A third possibility is that rapid particle loss to the auroral ionosphere caused by wave-particle interactions is occurring.

4. Intervals of geomagnetic quiet are associated with the far trailing portion of corotating streams where $|B|$ is low $(<3 \mathrm{nT})$ and there is an absence of Alfvenic fluctuations.

5. The physical mechanism for the "initial" (positive Dst) phases of magnetic storms is considerably different from that for fast ejecta events. For corotating streams it is the HCS plasma sheet and the stream-stream compressive effects that lead to the high solar wind plasma densities. These density enhancements can occur well ahead of high $|B|$ intensities, and thus long. duration, purely positive $D s t$ events result. Although the solar wind speeds in these regions are generally quite low, $-300-350$ $\mathrm{km} \mathrm{s}^{-1}$, the densities are often an order of magnitude larger than average values, allowing significant ram pressure increases and the storm initial phase.

6. The three major ( $D s t \leq-100 \mathrm{nT}$ ) magnetic storms that occurred in 1974 were not created by the corotating streams. All three were associated with moderate speed streams led by fastforward shocks. The streams were not particularly notable (the highest had a speed of $\sim 600 \mathrm{~km} \mathrm{~s}^{-1}$ ), and all were located near the HCS crossings. One speculation that we propose is that these relatively smallish events might be related to the coronal hole expansion, i.e., the opening of additional magnetic flux in the corona. It was not possible to find any (small) solar flares $x$ prominence eruptions associated with the interplanetary events.

7. Reverse shocks are present in corotating streams at 1 AU about $-20 \%$ of the time. They are not accompanied by forward shocks. If the prior fields had a southward direction, the decrease in magnetic field magnitude that occurs with a reverse shock 


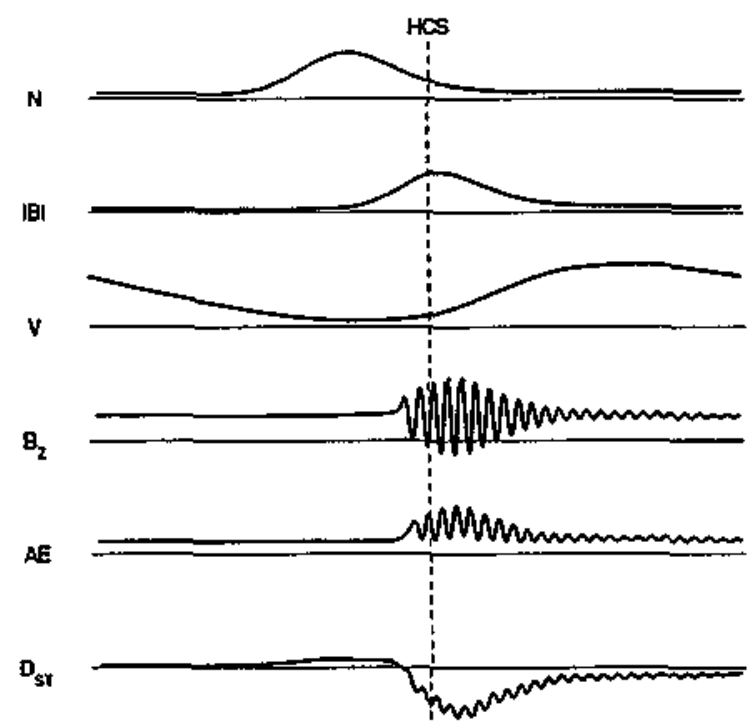

Figure 12. A schematic of the solar wind causes of geomagnetic quiet, small to moderate magnetic storms, and HILDCAAs during the descending phase of the solar cycle.

crossing (from downstream to upstream in time) causes the onset of the storm recovery phase.

8. The causes of the $B_{s}$ events responsible for the A, B, and C magnetic storms were shock compression of preexisting southward $B_{z}$ for the two largest storm events and $B_{s}$ within a magnetic cloud for the weakest event.

A schematic of geomagnetic active and geomagnetic quiet intervals summarizing the above results is given in Figure 12. Region 1 is the distant trailing portion of a high-speed stream. The speed is relatively low and is decreasing with time. The magnetic field magnitude is low and devoid of large-amplitude Alfvén waves. This is a region where $A E$ is lowest (other than times when $\boldsymbol{B}_{2}$ is northward for Jong intervals of time; see Tsurutani and Gonzalez [1995b]. In region 2 the plasma density is high owing to the presence of naturally occurring high densities near the HCS (the HCS plasma sheet) and also to stream-stream compressive effects. This region, when incident upon the magnetosphere, leadls to positive $D s t$ values. After the passage of this density enhancement, if the subsequent IMF has significant southward components causing a storm main phase, then the prior positive Dst will appear as a storm initial phase (without a sudden commencement). If there are no trailing southwardly directed fields, the former will only be a positive Dst event. Region 3 contains the high-intensity magnetic fields associated with streamstream compressive effects. Southward deflections of the IMF lead to small or moderate magnetic storms. Note that at $1 \mathrm{AU}$ this high field region is located at the beginning of the gradient of the high-speed stream and is not near the region where the gradient or velocity is the highest. This is considerably different from what happens with storms that occur during solar maximum (for shock compression of $B_{S}$ this occurs at high-speeds just behind the forward shock; for magnetic clouds this occurs near the peak ejecta speeds). Because the fields in the interaction region are highly fluctuating, the storm main phases are also highly irregular in profile. Region 4, at the peak and trailing portions of the highspeed stream, is characterized by Alfvénic fluctuations in $B_{z}$ and concomitant continuous auroral ativity (HILDCAAS). The $A E$ intensity is highest near the peak of the stream and decreases with decreasing solar wind speed and Alfvén wave amplitudes. Recent Ulysses high-latitude results have given us additional information which help put these in-ecliptic observations/results into context. Phillips et al. [1994] have shown that the solar wind associated with polar coronal holes has constant speeds near $750-800 \mathrm{~km} \mathrm{~s}^{-1}$. Tsurutani et al. [1994] and Smith et al. [1995] have shown that Alfvén fluctuations within such streams have continuous transverse fluctuations with $\Delta B /|B| \sim 1$ to 2 . Thus the most intense HILDCAAs are associated with cases in which the coronal hole stream proper impinges on the magnetosphere. Trailing portions of the streams (rarefaction regions) have lower Alfvenic wave amplitudes and thus are less geoeffective, consistent with the above observations.

Why does the idea of 27-day recurring magnetic storms persist? At the time of the Chapman and Bartels [1940] book, only midlatitude indices such as ap were available. From these indices, one could not tell whether the geomagnetic activity was due to storms or substorms. From this study we clearly show that both are present. However, we note that the dominant cause of ap periodicity is the long-duration auroral activity (HILDCAAs) throughout the corotating stream.

Acknowledgments. Portions of this work were done at the Jet Propulsion Laboratory, California Institute of Technology, Pasadena, under contract with the National Aeronautics and Space Administration. We wish to acknowledge helpful scientific discussions with E. J. Smith. This paper was submitted simultaneously with that of Crooker and Cliver $(99,23383,1994)$. The Editors (B. Hultquist and T. Gombosi), who have read both, feel that they should be viewed as complementary in nature. The authors would like to thank the referees for their constructive comments.

The Edjtor thasks two referees for their assistance in evaluating this paper.

\section{References}

Bartels, J., Some problems of terrestrial magnetism and electricity in Terrestrial Magnetism and Atmospheric Electriciry, edited by J. A. Flemming, p. 385, McGraw-Hill, New York, 1938.

Belcher, I. W., and L. Davis Jr., Large-amplitude Alfvén waves in the interplanetary medium, 2, J. Geophys. Res., 76, 3534, 1971.

Borrini, G., J. T. Gosling, S. J. Bame, W. C. Feldman, and J. M. Wilcox, Solar wind helium and hydrogen structure near the heliosphere current sheet: A signal of coronal structures at I AU, J. Geophys. Res., 86 . 4565,1981 .

Burlaga, L. F., and R. P. Lepping, The causes of recurtent geomagnetic storts, Planet. Space Sci., 25, 1151, 1977.

Burlaga, L. F., K. W. Behannon, S. F. Hansen, G. W. Pneumann, and W. C. Feldman, Sources of magnetic fields in recurrent interplanetary streams, J. Geophys. Res., 83, 4177, 1978.

Burlaga, L., E. Sittler, F. Mariani, and R. Schwenn, Magnetic loop behind an interplanetary shock: Voyagers, Helios, and IMP 8 observations, $J$. Geophys. Res., 86, 6673, 1981.

Burlaga, L. F., K. W. Behannon, and L. W. Klein, Compound streams. magnetic clouds, and major geomagnetic storms, J. Geophys. Res., 92, 5725, 1987

Chapman, S., and J. Bartels, Geomagnetism. vol. 1, chap. XII, 396, Clarendon, Oxford, England, 1940

Crooker, N. U., E. W. Cliver, and B. T. Tsurutani, The semiannual variation of great geomagnetic storms and the postshock RussellMcPherron effect preceding coronal mass ejecta, Geophys. Res. Lest. 19. 429, 1992a. 


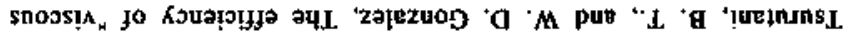

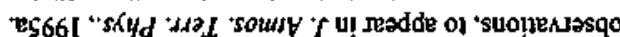

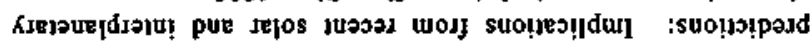

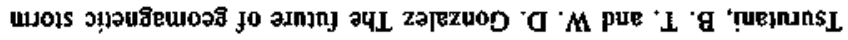

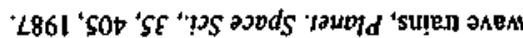

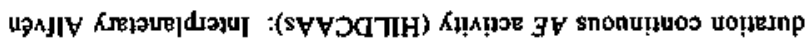

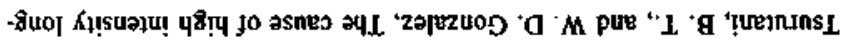

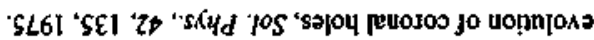

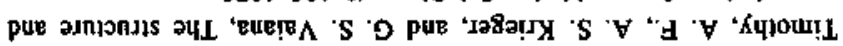

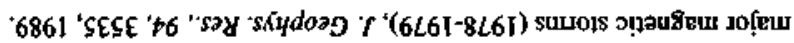

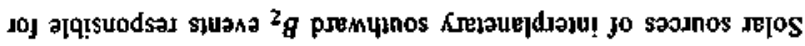

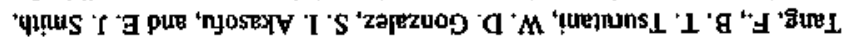

'\$661 '\$91 '9l "say әә0dS '

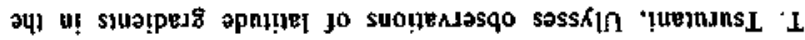

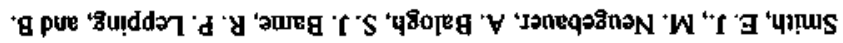

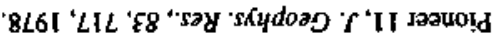

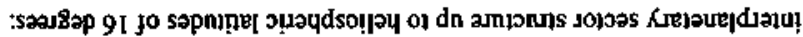

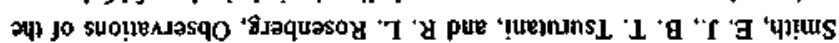

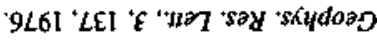

'Il pue ol ssaauoti : :

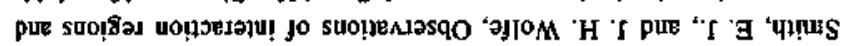

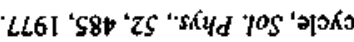

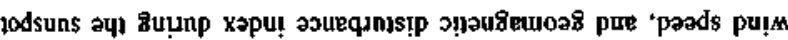

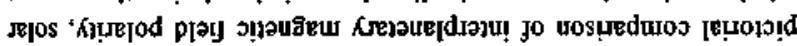
$\checkmark$ '

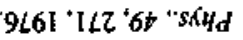

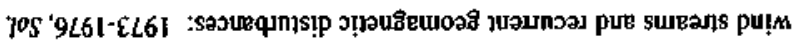

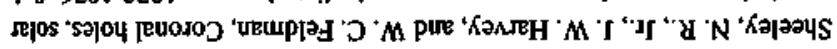

$996 \mathrm{I}$

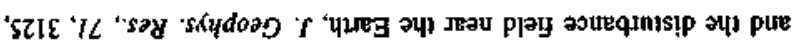

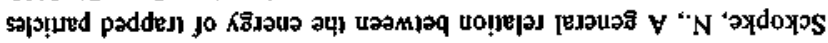

'Z66l 'ןlzedg 'opned oes 'sodure

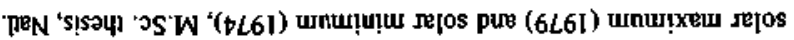

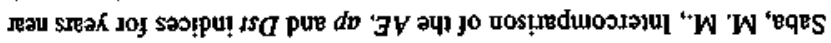

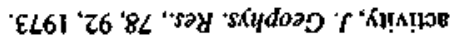

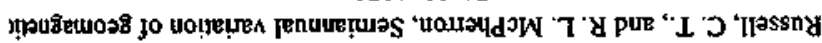

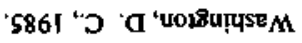

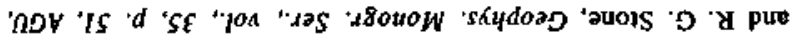

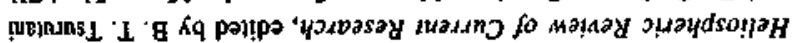

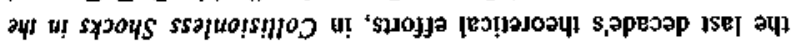

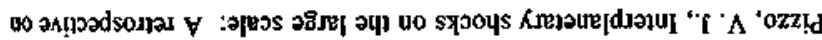
t66I

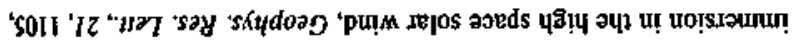

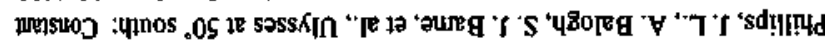
'996I '69to ' IL "say ssydoas of "puim

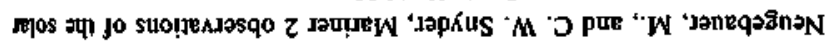
t

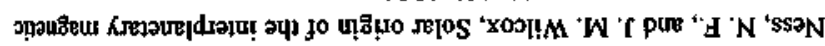

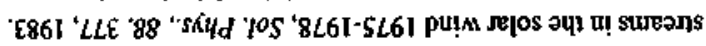

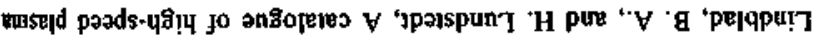

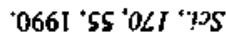

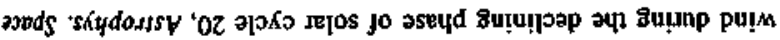

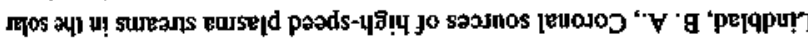
$\rightarrow<61$

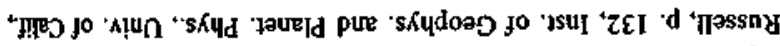

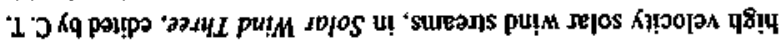

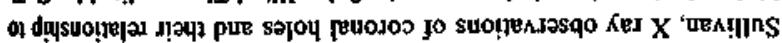

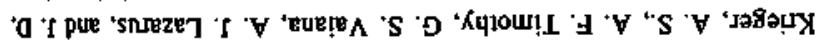
'EL61's0S

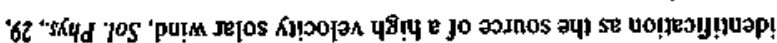

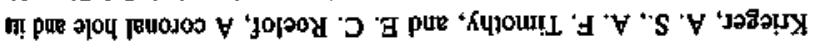

'Z861 'E19" $\angle 8$ "say skydoas

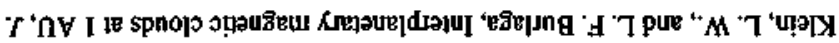

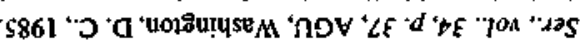

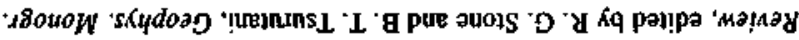

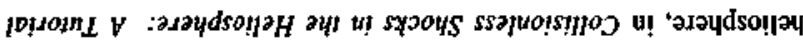

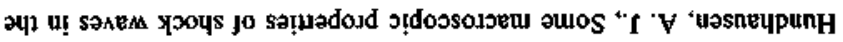
'EL6I 'seoz' $8 \angle$ "say skydozo of 'nY

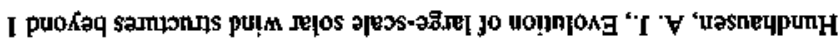

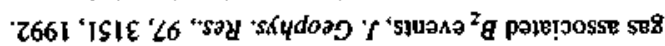

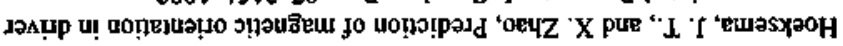

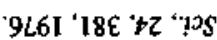

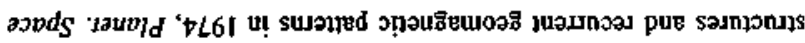

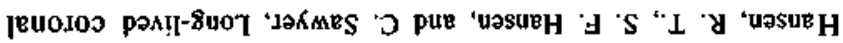

$166 \mathrm{I}$

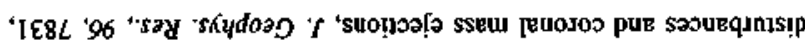

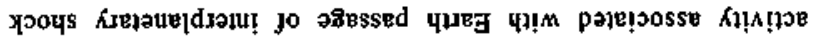

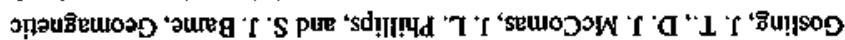
0661

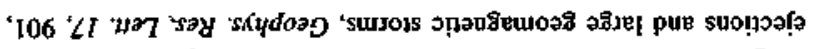

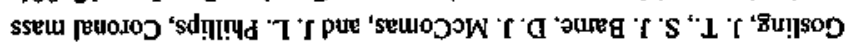

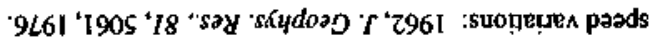

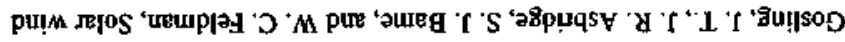

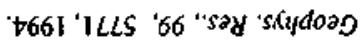

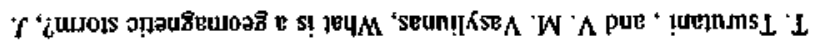

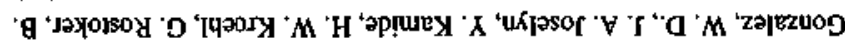

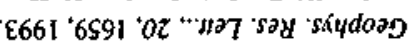

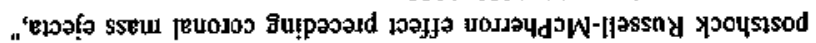

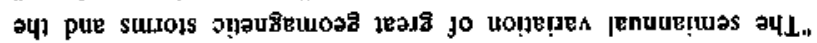

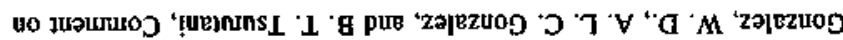

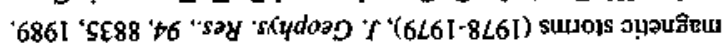

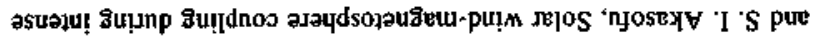

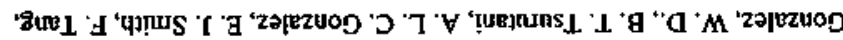
t66I '

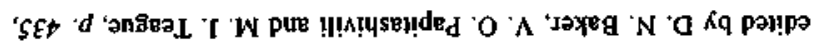

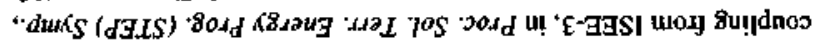

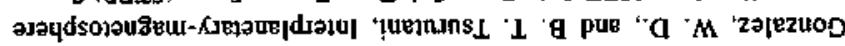

Z66I 'NOX MaN

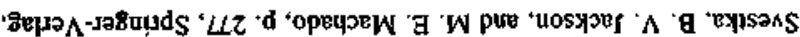

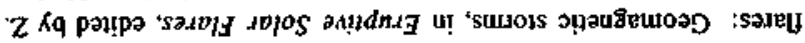

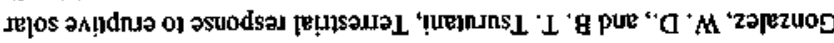

$\angle 861^{\circ} 101 \mathrm{I}$

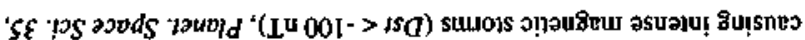

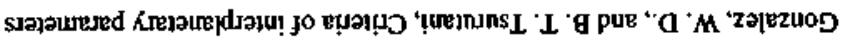

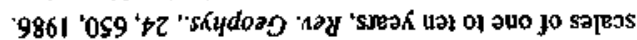

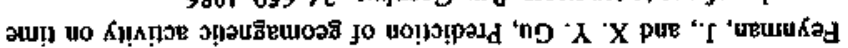

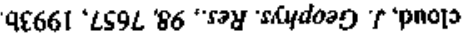

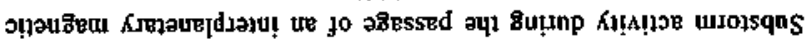

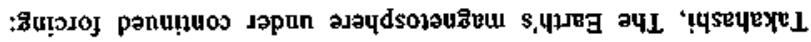

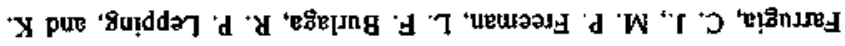
'e $\varepsilon 661$

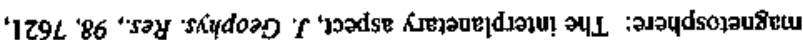

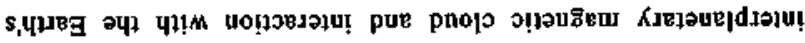

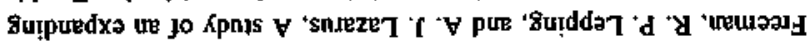

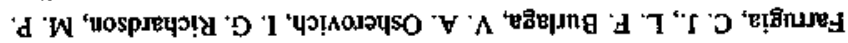
[ [961'Lt'9 " $\mathrm{m}$ '

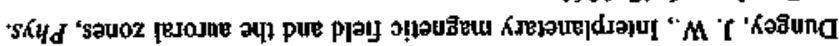

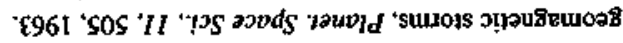

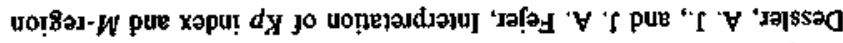

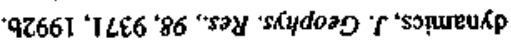

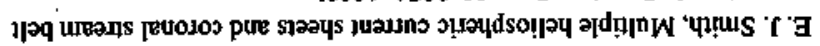

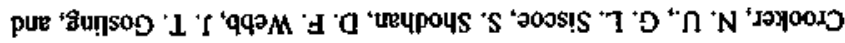


interaction" between the solar wind and the magnetosphere during intense northward IMF events, Geophys. Res. Lett, 22, 663, $1995 \mathrm{~b}$.

Isurutani, B. T., E. J. Smith, K. R. Pyle, and J. A. Simpson, Energetic protons accelerated at corotating shocks: Pioneer 10 and 11 observations from 1 to $6 \mathrm{AU}$, J. Geophys. Res., 87, 7389, 1982.

Tsurulani, B. T., W. D. Gonzalez. F. Tang, S. J. Akasofu, and E. J. Smith, Origin of interplanetary southward magnetic fields responsible for major magnetic storms near solar maximum (1978-1979), J. Geophys. Res., 93, 8519, 1988.

Tsurutani, B. T., T. Gould, B. E. Goldstein, W. D. Gonzalez, and M. Sugiura, Interplanetary Alfvér waves and auroral (substorm) activity: IMP 8, J. Geophys. Res.. 95, 2241, 1990.

Tsurutani, B. T.. W. D. Gonzalez, F. Tang, and Y. T. Lee, Great magnetic stoms, Geophys. Res. Lett. 19, 73, 1992.

Tsurutani, B. T., C. M. Ho, E. J. Smith, M. Neugebauer, B. E. Goldstein, I. S. Mok, J. K. Arballo, A. Balogh, D. J. Southwood, and W. C. Feldman. The relationship between interplanetary discontinuities and Alfvénic waves: Ulysses observations, Geophys. Res. Lelt. 21, 2267. 1994.

Wilcox, J. M., and N. F. Ness, Quasi-stationary corotating structure in the interplanetary medium, J. Geophys. Res., 70, 5793, 1965.

Winterhahter, D., E. J. Smith, M. E. Burton, N. Murphy, and D. J.
McComas, The heliospheric plasma sheet, J. Geophys. Res.. 99. 6667, 1994.

Zwickl, R. D., J. R. Asbridge, S. J. Bame, W. C. Feldman, J. T. Gosling. and E. J. Smith, Plasma properties of driver gas following interplanetary shocks observed by ISEE-3, in Solar Wind Five, NASA Conf. Publ., CP-2280, 711, 1983.

J. K. Arballo and B. T. Tsurutani, Jet Propulsion Laboratory. Califomia Institute of Technology, MS 169-506, 4800 Oak Grove Drive, Pasadena, CA 91109. (e-mail: jarballo@jplsp.jpl.nasa.gov; btsurutani@jplsp.jpl.nasagov)

A. L. C. Gonzalez and W. D. Gonzalez, Instituto National Pesquisas Espaciais (INPE), Sao Jose dos Campos, Sao Paulo, Brazil. (e-mail: gonzalez@das.inpe.br)

M. Okada, National Institute of Polar Research, Tokyo, Japan. (e-mail: mokada@hp9000.nipr.ac.jp)

F. Tang, California Institute of Technology, Pasadena, CA 91125. (email: ft(

(Received Febriary 25, 1994; revised May 5, 1995;

accepted May 5, 1995.) 